\title{
Axisymmetric Hadley Cell Theory with a Fixed Tropopause Temperature Rather than Height
}

\author{
SPENCER A. HiLl ${ }^{\mathrm{a}}$ \\ Division of Geological and Planetary Sciences, California Institute of Technology, Pasadena, and \\ Department of Earth, Planetary, and Space Sciences, University of California, Los Angeles, Los Angeles, California
}

SIMONA BORDONI

Division of Geological and Planetary Sciences, California Institute of Technology, Pasadena, California, and Department of Civil, Environmental and Mechanical Engineering (DICAM), University of Trento, Trento, Italy

\section{JONATHAN L. MITCHELL}

Department of Earth, Planetary, and Space Sciences, and Department of Atmospheric and Oceanic Sciences, University of California, Los Angeles, Los Angeles, California

(Manuscript received 24 June 2019, in final form 7 February 2020)

\begin{abstract}
Axisymmetric Hadley cell theory has traditionally assumed that the tropopause height $\left(H_{\mathrm{t}}\right)$ is uniform and unchanged from its radiative-convective equilibrium (RCE) value by the cells' emergence. Recent studies suggest that the tropopause temperature $\left(T_{\mathrm{t}}\right)$, not height, is nearly invariant in RCE, which would require appreciable meridional variations in $H_{\mathrm{t}}$. Here, we derive modified expressions of axisymmetric theory by assuming a fixed $T_{\mathrm{t}}$ and compare the results to their fixed- $H_{\mathrm{t}}$ counterparts. If $T_{\mathrm{t}}$ and the depth-averaged lapse rate are meridionally uniform, then at each latitude $H_{\mathrm{t}}$ varies linearly with the local surface temperature, altering the diagnosed gradient-balanced zonal wind at the tropopause appreciably (up to tens of meters per second) but the minimal Hadley cell extent predicted by Hide's theorem only weakly $\left(\Sigma^{\circ}\right)$ under standard annual-mean and solsticial forcings. A uniform $T_{\mathrm{t}}$ alters the thermal field required to generate an angularmomentum-conserving Hadley circulation, but these changes and the resulting changes to the equal-area model solutions for the cell edges again are modest $(<10 \%)$. In numerical simulations of latitude-by-latitude RCE under annual-mean forcing using a single-column model, assuming a uniform $T_{\mathrm{t}}$ is reasonably accurate up to the midlatitudes, and the Hide's theorem metrics are again qualitatively insensitive to the tropopause definition. However imperfectly axisymmetric theory portrays the Hadley cells in Earth's macroturbulent atmosphere, evidently its treatment of the tropopause is not an important error source.
\end{abstract}

\section{Introduction}

Absent any large-scale circulation, local radiativeconvective equilibrium (RCE) would necessarily prevail at each latitude: the large-scale meridional and vertical velocities vanishing, the lapse rate nearly adiabatic from the surface to the tropopause, and the interplay between local radiative forcing and convection generating equilibrium temperature and zonal wind fields in gradient

\footnotetext{
${ }^{a}$ Current affiliation: Lamont-Doherty Earth Observatory, Columbia University, New York, New York.
}

Corresponding author: Spencer Hill, shill@ldeo.columbia.edu balance. Provided zonal flow is weak at the surface, the tropopause height then controls the maximum magnitude of the zonal wind within the troposphere at each latitude. Via Hide's theorem, the resulting absolute angular momentum and absolute vorticity fields determine the emergence (Hide 1969; Schneider 1977; Plumb and Hou 1992) and minimal extent (Held and Hou 1980; Hill et al. 2019) of the Hadley cells in axisymmetric and zonally varying (Emanuel 1995) atmospheres.

The original expressions of Hide's theorem and of the angular-momentum-conserving (AMC) model of the Hadley cells (Schneider 1977; Held and Hou 1980) were derived in a dry, Boussinesq framework in which the tropopause height $H_{\mathrm{t}}$ is assumed uniform in latitude and, 
if a Hadley circulation emerges, to be unaltered from its RCE value. This includes the equal-area model for the cell edge locations that combines the AMC assumption with assumptions of continuity in depth-averaged potential temperature and energy conservation within each cell (Held and Hou 1980; Schneider 1987; Lindzen and Hou 1988). In the adaptation of axisymmetric theory to moist atmospheres obeying convective quasi equilibrium (CQE; Emanuel et al. 1994; Emanuel 1995), the tropopause height — which appears in temperature coordinates as the difference between the surface and tropopause temperatures, $T_{\mathrm{s}}-T_{\mathrm{t}}$-is not assumed uniform in the expression of Hide's theorem [(10) of Emanuel 1995] but is in deriving the AMC, or "critical," subcloud equivalent potential temperature field [(11) of Emanuel 1995].

That assuming a fixed tropopause height may be problematic can be seen from the hypsometric equation applied at each latitude over the local depth of the troposphere: $H_{\mathrm{t}}=\left(R_{\mathrm{d}} / g\right) \hat{T} \ln \left(p_{\mathrm{s}} / p_{\mathrm{t}}\right)$, where $R_{\mathrm{d}}$ is the atmospheric gas constant, $g$ is gravity, and $\hat{T}$ is temperature averaged in log pressure from the surface with pressure $p_{\mathrm{s}}$ to the tropopause with pressure $p_{\mathrm{t}}$. Neglecting surface pressure variations, a given value of $H_{\mathrm{t}}$ will occur at larger $p_{\mathrm{t}}$ values in warmer columns compared to cooler columns-opposite to both simulations of latitude-bylatitude RCE to be presented below and to observations, ${ }^{1}$ wherein the tropopause pressure is lowest (and height is highest) in the warmest columns (e.g., Held 1982; Hu and Vallis 2019).

Recent work based on RCE simulations in a doubly periodic, cloud-resolving model suggests that the tropopause temperature $T_{\mathrm{t}}$, rather than height, is nearly climate-state invariant averaged over the domain (Seeley et al. 2019b), refining (Seeley et al. 2019a) previous arguments that cloud anvils form at a climate-state-invariant temperature both in the tropics (Hartmann and Larson 2002) and extratropics (Thompson et al. 2017, 2019). ${ }^{2}$ The tropopause temperature is also invariant in simple, singlecolumn theoretical models for the tropopause height that assume gray radiative transfer and an optically thin stratosphere (e.g., Vallis et al. 2015; Hu and Vallis 2019). And a fixed $T_{\mathrm{t}}$ is effectively assumed (via an isothermal, 200-K stratosphere) in widely used Newtonian cooling profiles including the canonical Held and Suarez (1994)

\footnotetext{
${ }^{1}$ The observed tropopause structure reflects, in addition to these radiative-convective influences, dynamical and radiative influences induced by the large-scale circulation, making this comparison with pure RCE imperfect.

${ }^{2}$ Specifically, in doubly periodic, fixed-SST, cloud-resolvingmodel simulations with uniform, prescribed sea surface temperatures varied from 260 to $310 \mathrm{~K}$, the domain-mean tropopause temperature varies by $\lesssim 5 \mathrm{~K}$ (Seeley et al. 2019b).
}

forcing and that of the Schneider (2004) idealized, dry GCM used in many recent studies of the Hadley cells (e.g.,Walker and Schneider 2005, 2006; Schneider and Bordoni 2008; Hill et al. 2019).

Absent compensating lapse-rate variations, a fixed tropopause temperature would cause $H_{\mathrm{t}}$ to vary with surface temperature: given a lapse rate $\Gamma \equiv \partial_{z} T$ vertically averaged (in $z$ coordinates) from the surface to the tropopause, $\hat{\Gamma}$, the tropopause height must satisfy

$$
H_{\mathrm{t}}=\frac{T_{\mathrm{s}}-T_{\mathrm{t}}}{\hat{\Gamma}}
$$

If $T_{\mathrm{t}}$ and $\hat{\Gamma}$ are fixed, $H_{\mathrm{t}}$ varies linearly with $T_{\mathrm{s}}$-rising on Earth, for example, by approximately $1 \mathrm{~km}$ per $10 \mathrm{~K}$ of surface warming in dry-adiabatic columns or per $\sim 6.5 \mathrm{~K}$ in moist-adiabatic columns. Insofar as $\hat{\Gamma}$ and $T_{\mathrm{t}}$ are meridionally uniform, (1) applied at each latitude immediately resolves the aforementioned problem arising from a uniform tropopause height: $T_{\mathrm{s}}-T_{\mathrm{t}}$, and therefore $H_{\mathrm{t}}$, must increase with $T_{\mathrm{s}}$.

While the original, fixed- $H_{\mathrm{t}}$ theories have been remarkably successful for Hadley cells on Earth and other planetary bodies at the conceptual to qualitative levels, it remains possible that (and, to our knowledge, unexamined whether) appreciable quantitative or even qualitative changes to their results emerge when meridional variations in $H_{\mathrm{t}}$ are accounted for. Fang and Tung (1994) compare analytical Hadley cell solutions attained with a fixed $H_{\mathrm{t}}$ with or without the tropopause temperature assumed fixed in their linear, viscous model, but this viscous regime is inappropriate for Earth's nearly inviscid free troposphere. Zalucha et al. (2010) incorporate meridional variations in surface height into the equal-area model, motivated by the mean downward surface slope from the south pole to the low latitudes on Mars (Richardson and Wilson 2002).

At the same time, Singh (2019) demonstrates that $T_{\mathrm{s}}-T_{\mathrm{t}}$, not $T_{\mathrm{t}}$, is nearly meridionally uniform in a GCM simulation of latitude-by-latitude RCE [his Fig. 5(b)]. So it remains necessary to assess the validity of a fixed $T_{\mathrm{t}}$ in simulations of latitude-by-latitude RCE, and-to solidify the link between simulation and theory-how usefully simple semianalytical approximations and the analytical forcing profiles underlying classical dry, Boussinesq, axisymmetric theory (Held and Hou 1980; Lindzen and Hou 1988) can approximate those moist, numerically simulated RCE solutions. ${ }^{3}$

\footnotetext{
${ }^{3}$ Singh (2019) also demonstrates that $T_{\mathrm{s}}-T_{\mathrm{t}}$ at the crossequatorial Hadley cell edge is nearly climate-state invariant across dynamically equilibrated simulations with a wide range of planetary rotation rates (his Fig. 9).
} 
These considerations motivate the present study. We begin by demonstrating that, based on (1) and provided $T_{\mathrm{t}}$ and $\hat{\Gamma}$ are meridionally uniform, meridional tropopause height variations can indeed be large in the latitude-by-latitude RCE state generated under both annual-mean and more solstice-like forcings (section 2). We then derive a fixed- $T_{\mathrm{t}}$ solution for the gradientbalanced zonal wind at the tropopause in these RCE states, compare it to its fixed- $H_{\mathrm{t}}$ counterpart (section 3 ), and investigate the resulting influences on the three conditions (Adam and Paldor 2010; Hill et al. 2019) of Hide's theorem for the emergence and minimal extent of the Hadley circulation (section 4). In a similar progression, we then derive fixed- $T_{\mathrm{t}}$ forms of the AMC (i.e., critical) depth-averaged potential temperature distribution (section 5) and explore the resulting impacts on the equal-area model (section 6). We then generate numerical, annual-mean, latitude-by-latitude RCE solutions via single-column model simulations to assess whether a meridionally uniform $T_{\mathrm{t}}$ is plausible, how sensitive the gradient wind at the tropopause and the Hide's theorem metrics are to the tropopause definition, and how accurately the numerical results can be captured by simple analytical and semianalytical approximations (section 7).

As fully summarized in section 8 , we find overall that the results of axisymmetric theory are qualitatively insensitive to how the tropopause is treated. This should add to the community's confidence in the original axisymmetric theories (within those parameter regimes in which they are justifiable in the first place), one of several implications we discuss in section 9.

\section{Effect of a fixed $T_{t}$ on the tropopause height in latitude-by-latitude RCE}

Figure 1 shows $H_{\mathrm{t}}$ diagnosed using (1) assuming $T_{\mathrm{t}}=200 \mathrm{~K}$, a meridionally and vertically uniform lapse rate of $\Gamma=\Gamma_{\mathrm{d}}$ with $\Gamma_{\mathrm{d}}=g / c_{p}$ the dry adiabatic lapse rate with gravity $g$ and specific heat of air at constant pressure $c_{p}$, and a vertically averaged potential temperature distribution $\hat{\theta} \equiv\left(1 / H_{\mathrm{t}}\right) \int_{0}^{H_{\mathrm{t}}} \theta \mathrm{d} z$ given by the canonical vertically averaged forcing of Lindzen and Hou (1988):

$$
\frac{\hat{\theta}_{\mathrm{rce}}}{\theta_{0}}=1+\frac{\Delta_{\mathrm{h}}}{3}\left[1-3\left(\sin \varphi-\sin \varphi_{\mathrm{m}}\right)^{2}\right] .
$$

In (2), $\hat{\theta}_{\text {rce }}$ maximizes at the latitude $\varphi_{\mathrm{m}}$, which we set to either $0^{\circ}, 6^{\circ}$, or $23.5^{\circ} \mathrm{N}, \Delta_{\mathrm{h}}$ is an imposed fractional planetary-scale temperature contrast set to $1 / 6$, and the Boussinesq reference potential temperature $\theta_{0}$ is set to $290 \mathrm{~K}$.

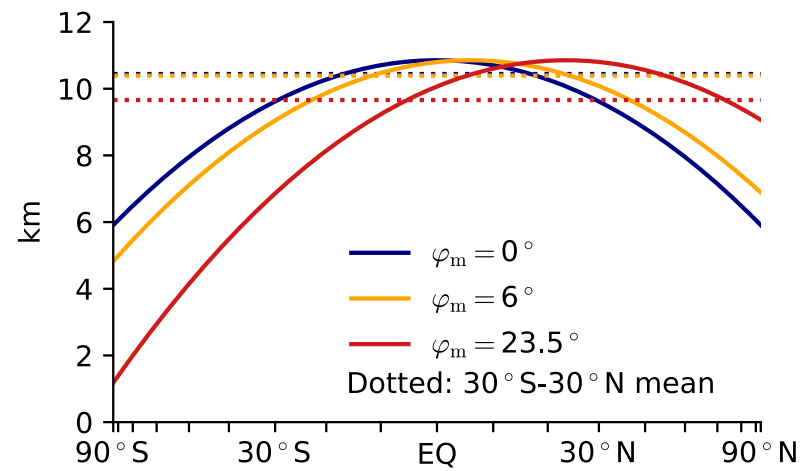

FIG. 1. Solid curves show tropopause height in the RCE state given by (2) with the forcing maximum latitude value $\varphi_{\mathrm{m}}$ according to the legend, assuming a uniform tropopause temperature of $200 \mathrm{~K}$ and a dry adiabatic lapse rate. Dotted lines show the fixed-height tropopause to which we compare the fixed-temperature results, defined as the tropical-mean $\left(30^{\circ} \mathrm{S}-30^{\circ} \mathrm{N}\right.$, area-weighted average) value of the solid curve with the corresponding color. Note that the horizontal-axis spacing is linear in the sine of latitude here and in all subsequent figures unless otherwise noted in the corresponding caption.

In the annual-mean case of $\varphi_{\mathrm{m}}=0^{\circ}$, the RCE tropopause height is $\sim 11 \mathrm{~km}$ at the equator and decreases to $\sim 9 \mathrm{~km}$ at $\pm 30^{\circ}$ and $\sim 6 \mathrm{~km}$ at the poles. As the forcing maximum latitude is moved into the Northern Hemisphere, by construction the tropopause height at $\varphi_{\mathrm{m}}$ remains $\sim 11 \mathrm{~km}$, but the tropopause drops to successively lower values in the southern, winter hemisphere. The $\varphi_{\mathrm{m}}=23.5^{\circ}$ case reveals the oversimplicity of this expression sufficiently far from the forcing maximum, with $H_{\mathrm{t}}$ dropping below $2 \mathrm{~km}$ at the poles; for $\varphi_{\mathrm{m}}$ sufficiently farther poleward (not shown), $T_{\mathrm{s}}$ drops below $T_{\mathrm{t}}$ near the winter pole, yielding the fallacious prediction of a vanishing or negative tropopause height.

Restricting to low to midlatitudes where the results are more reasonable and of more relevance to the Hadley cells, $H_{\mathrm{t}}$ varies meridionally by up to $\sim 5 \mathrm{~km}$, that is, by $\sim 40 \%$ - variations sufficiently large to motivate examining their influence on the corresponding gradient-balanced zonal wind field at the tropopause.

\section{Effect of a fixed $T_{t}$ on the gradient-balanced zonal wind at the tropopause}

When integrated in height from the surface to the tropopause and assuming negligible surface zonal wind, gradient balance in a Boussinesq atmosphere may be written

$$
2 \Omega \sin \varphi u_{\mathrm{t}}+\frac{\tan \varphi}{a} u_{\mathrm{t}}^{2}=-\frac{g}{a \theta_{0}} \int_{0}^{H_{\mathrm{t}}(\varphi)} \frac{\partial \theta}{\partial \varphi} \mathrm{d} z,
$$


where $\Omega$ is planetary rotation rate, $\varphi$ is latitude, $u_{\mathrm{t}}$ is zonal wind at the tropopause, $a$ is planetary radius, and $\theta$ is the perturbation potential temperature. ${ }^{4}$

In the presence of nonzero $\partial_{\varphi} H_{\mathrm{t}}$, (3) can still be solved as standard for $u_{\mathrm{t}}$ provided that the stratification is dry adiabatic. Otherwise, $\theta$ and $\partial_{\varphi} \theta$ are not generally uniform in height, and applying the Leibniz integral rule generates an additional term. For the quantities of interest regarding axisymmetric Hadley cell theory, the presence of this term complicates all subsequent derivations without adding physical insight. Similarly, in the moist, CQE system, though by construction the stratification is moist adiabatic and this extra term therefore absent, the presence of moisture complicates the derivations in ways that do not add physical insight as regards our present focus. Moreover, the influence of a fixed $T_{\mathrm{t}}$ on the fields of interest is similar in all three cases (Boussinesq dry adiabatic, Boussinesq non-dry adiabatic, and CQE; not shown). Accordingly, in the main text we focus on the dry adiabatic case, relegating derivations and results for the dry-stably stratified Boussinesq case to appendix $\mathrm{A}$ and for the CQE case to appendix B.

Restricting then to the general Boussinesq, dry-adiabatic case in the presence of meridional $H_{\mathrm{t}}$ variations, solving (3) yields

$$
u_{\mathrm{t}}=\Omega a \cos \varphi\left[\sqrt{1-\frac{1}{\cos \varphi \sin \varphi} \frac{g H_{\mathrm{t}}}{\Omega^{2} a^{2} \theta_{0}} \frac{\partial \hat{\theta}}{\partial \varphi}}-1\right]
$$

Applied to each latitude of the hypothetical RCE state and using (1), this becomes an expression for the RCE gradient-balanced zonal wind at the tropopause,

$$
u_{\mathrm{t}, \mathrm{rce}}=\Omega a \cos \varphi\left[\sqrt{1-\frac{1}{\cos \varphi \sin \varphi} \frac{c_{p}\left(T_{\mathrm{s}}-T_{\mathrm{t}}\right)}{\Omega^{2} a^{2} \theta_{0}} \frac{\partial T_{\mathrm{s}}}{\partial \varphi}}-1\right]
$$

\footnotetext{
${ }^{4}$ The perturbation potential temperature is in hydrostatic balance with a perturbation pressure field $p^{\prime}$, according to $\partial_{z} \Phi=g \theta / \theta_{0}$, where $\Phi=p^{\prime} / p_{0}$ and $\rho_{0}$ is the reference density [note that Hill et al. (2019) incorrectly refer to $\Phi$ as "geopotential"]. Lindzen and Hou (1988) and Held and Hou (1980) define $\hat{\theta}_{\text {rce }}$ [in (2)] as the RCE full depth-averaged potential temperature; elsewhere, those authors use $\theta$ and $\hat{\theta}$ to refer either to the full or perturbation (i.e., the full potential temperature minus $\theta_{0}$ ) potential temperature depending on the context. This casualness is not problematic in those studies or the present one, because all potential temperature fields only appear as derivatives or differences for which the use of full or perturbation quantities is irrelevant. As such, henceforth we refer without confusion to $\theta$ as simply potential temperature and $\hat{\theta}$ as simply depth-averaged potential temperature.
}

which in fact holds whether or not $T_{\mathrm{t}}$ is assumed uniform.

Comparing fixed- $H_{\mathrm{t}}$ and fixed- $T_{\mathrm{t}}$ results of (5) requires choosing values for each version's fixed parameters. In all that follows, for the fixed- $T_{\mathrm{t}}$ value we use $200 \mathrm{~K}$, and for the fixed- $H_{\mathrm{t}}$ value we use the tropicalmean $\left(30^{\circ} \mathrm{S}-30^{\circ} \mathrm{N}\right.$, area-weighted) value of $H_{\mathrm{t}}$, denoted $\overline{H_{\mathrm{t}}}$, taken from the corresponding fixed- $T_{\mathrm{t}}$ calculation (cf. dotted lines in Fig. 1). This subjective choice was made to avoid artificially inflating the seeming influence of a fixed $T_{\mathrm{t}}$ within the latitudes of relevance to the Hadley cells. Corresponding to this $\overline{H_{\mathrm{t}}}$ value and the given $T_{\mathrm{t}}$ and $\hat{\Gamma}$ values is a characteristic surface temperature $\overline{T_{\mathrm{s}}}=T_{\mathrm{t}}+\hat{\Gamma} \overline{H_{\mathrm{t}}}$. The fixed- $T_{\mathrm{t}}$ calculation of $u_{\mathrm{t}, \text { rce }}$ then differs from its fixed- $H_{\mathrm{t}}$ counterpart at any latitude whose surface temperature differs from that tropicalmean value: where $T_{\mathrm{s}}>\overline{T_{\mathrm{s}}}$, the magnitude of the diagnosed $u_{\mathrm{t}}$ increases, and where $T_{\mathrm{s}}<\overline{T_{\mathrm{s}}}$, the magnitude of the diagnosed $u_{\mathrm{t}}$ decreases relative to the fixed-height calculation. Results are qualitatively insensitive to reasonable variations to this averaging domain (not shown).

Figure 2 a shows $u_{\mathrm{t} \text {,rce }}$ diagnosed using (4) with either $T_{\mathrm{t}}$ fixed to $200 \mathrm{~K}$ or $H_{\mathrm{t}}$ fixed as just described for $\varphi_{\mathrm{m}}$ equal to $0^{\circ}, 6^{\circ}$, or $23.5^{\circ} \mathrm{N}$. In the annual-mean-like case $\left(\varphi_{\mathrm{m}}=0^{\circ}\right)$, the tropopause's maximum height at the equator and its monotonic decrease toward either pole makes the fixed- $T_{\mathrm{t}}$-calculated $u_{\mathrm{t}, \text { rce }}$ more westerly than its fixed- $H_{\mathrm{t}}$ counterpart from the equator to $\sim 15^{\circ} \mathrm{S} / \mathrm{N}$, but only by $\$ 3 \mathrm{~m} \mathrm{~s}^{-1}$. Poleward thereof, the tropopause height is lower than its tropical-mean value, and this reduces $u_{\mathrm{t}, \mathrm{rce}}$ compared to the fixed- $H_{\mathrm{t}}$ formulation by up to $5 \mathrm{~m} \mathrm{~s}^{-1}$.

For the solstice-like cases $\left(\varphi_{\mathrm{m}}=6^{\circ}, 23.5^{\circ}\right)$, the relatively deep tropopause near $\varphi_{\mathrm{m}}$ slightly increases the magnitudes of both the easterlies on its equatorward side within the summer hemisphere and the westerlies on its poleward side. In the winter hemisphere, the westerlies are weakened more appreciably-up to $\sim 20 \mathrm{~m} \mathrm{~s}^{-1}$ in the midlatitudes and by $\sim 10 \mathrm{~m} \mathrm{~s}^{-1}$ in the subtropics for $\varphi_{\mathrm{m}}=23.5^{\circ}$-due to a comparatively low tropopause height locally.

\section{Effect of a fixed $T_{t}$ on the minimal Hadley cell extent based on Hide's theorem}

Given $u_{\mathrm{t}, \mathrm{rce}}$, any isolated extremum in the corresponding absolute angular momentum field, $M_{\mathrm{t}, \mathrm{rce}}=$ $a \cos \varphi\left(\Omega a \cos \varphi+u_{\mathrm{t}, \mathrm{rce}}\right)$, or (nearly equivalently) any sign change within either hemisphere in the corresponding absolute vorticity field, $\eta_{\mathrm{t}, \mathrm{rce}}=-\left(a^{2} \cos \varphi\right)^{-1} \partial_{\varphi} M_{\mathrm{t}, \mathrm{rce}}=f-$ $a^{-1} \partial_{\varphi} u_{\mathrm{t}, \text { rce }}$, is unattainable in a steady, axisymmetric atmosphere (Hide 1969; Schneider 1977; Plumb and Hou 1992). Therefore, a Hadley cell must emerge if the RCE state exhibits any of $M_{\mathrm{t}, \mathrm{rce}}>\Omega a^{2}, M_{\mathrm{t}, \mathrm{rce}}<0$, or $f \eta_{\mathrm{t}, \mathrm{rce}}<0$ 

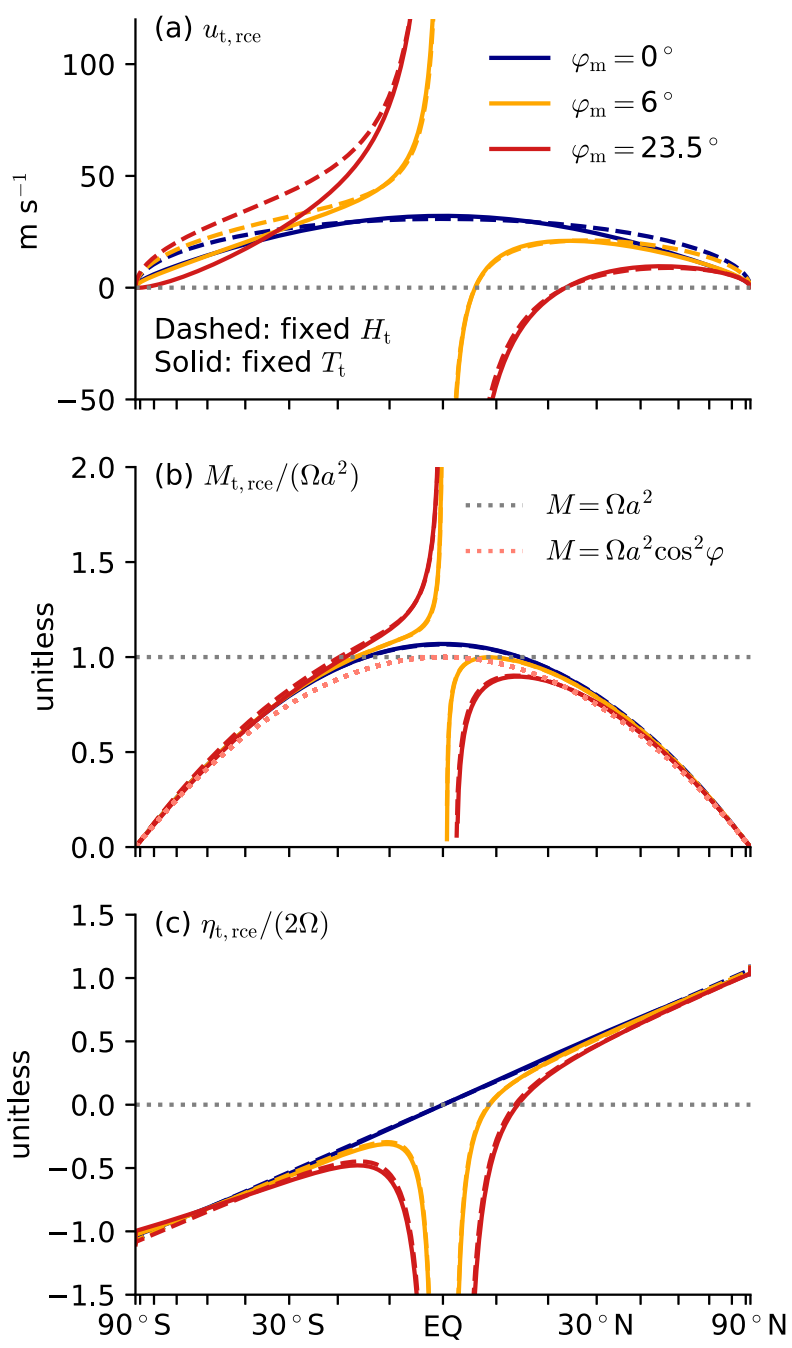

FIG. 2. At the local tropopause, (a) zonal wind $\left(\mathrm{m} \mathrm{s}^{-1}\right)$, (b) absolute angular momentum normalized by the equatorial planetary angular momentum, and (c) absolute vorticity normalized by $2 \Omega$, in the RCE state given by (2) with the forcing maximum latitude $\varphi_{\mathrm{m}}$ value according to the legend in (a). Dashed lines indicate the tropopause height is assumed constant; solid lines indicate the tropopause temperature is assumed constant. The fixed-height calculations use the $30^{\circ} \mathrm{S}-30^{\circ} \mathrm{N}$ average tropopause height listed in Table 1 from the corresponding fixed-temperature calculation (all of which set $T_{\mathrm{t}}=$ $200 \mathrm{~K}$ ). Overlaid in (b) are the equatorial planetary angular momentum as a dotted gray curve and the local planetary angular momentum as a dotted pink curve. Overlaid in (c) is the $\eta=0$ curve as a dotted gray line, which would correspond to uniform angular momentum, e.g., the dotted gray curve in (b).

at any latitude $\left(\Omega a^{2}\right.$ and 0 being the extremal planetary values of angular momentum, at the equator and either pole, respectively), and at the very least the resulting circulation must span all latitudes meeting any of these three conditions of Hide's theorem (Hill et al. 2019). For annual-mean-like forcing, only the $M_{\mathrm{t}, \text { rce }}>\Omega a^{2}$ condition is typically met; for solstice-like forcing, typically the $M_{\mathrm{t}, \mathrm{rce}}>\Omega a^{2}$ condition is met over some appreciable range of the winter hemisphere, the $M_{\mathrm{t}, \text { rce }}<0$ condition is met in a narrow range near the equator in the summer hemisphere (though under exotic forcings it can extend to the summer pole; cf. Hill et al. 2019), and the $f \eta_{\text {t,rce }}<0$ condition is met over some appreciable range poleward thereof, making where $\eta_{\mathrm{t} \text {,rce }}=0$ in the summer hemisphere a lower bound on how far the Hadley circulation extends into the summer hemisphere.

Directly at the equator, these three conditions serve purely as Hadley cell emergence indicators and are unaffected by meridional variations in $H_{\mathrm{t}}$ : an equatorial $\hat{\theta}_{\text {rce }}$ maximum still yields equatorial westerlies and thus $M_{\mathrm{t}, \text { rce }}>\Omega a^{2}$ locally, and a nonzero cross-equatorial $\hat{\theta}_{\text {rce }}$ gradient still cannot be balanced. But as indicators of minimal cell extent away from the equator, the $M_{\mathrm{t}, \text { rce }}>\Omega a^{2}, M_{\mathrm{t}, \text { rce }}<0$, and $f \eta_{\mathrm{t}, \mathrm{rce}}<0$ extents are all in principle altered by the tropopause treatment.

Figure 2b shows $M_{\mathrm{t}, \mathrm{rce}}$ computed for the same $\varphi_{\mathrm{m}}=$ $0^{\circ}, 6^{\circ}, 23.5^{\circ}$ cases discussed above, with either $H_{\mathrm{t}}$ or $T_{\mathrm{t}}$ fixed, and Table 1 shows the maximal poleward extent of each Hide's theorem metric. For $\varphi_{\mathrm{m}}=0^{\circ}$ and $\varphi_{\mathrm{m}}=6^{\circ}$, the $M_{\mathrm{t}, \text { rce }}>\Omega a^{2}$ extent is essentially unchanged by the fixed- $T_{\mathrm{t}}$ formulation. For $\varphi_{\mathrm{m}}=23.5^{\circ}$, the summer hemisphere westerlies are far enough poleward (where the planetary angular momentum values are lower) that neither the fixed- $H_{\mathrm{t}}$ nor fixed- $T_{\mathrm{t}}$ calculations of $M_{\mathrm{t}, \mathrm{rce}}$ exceed $\Omega a^{2}$ in the summer hemisphere. In the winter hemisphere the $M_{\mathrm{t}, \mathrm{rce}}>\Omega a^{2}$ extent contracts equatorward by a more appreciable $1.2^{\circ}$.

Figure $2 \mathrm{c}$ shows the corresponding calculations of $\eta_{\mathrm{t} \text {,rce }}$, and Table 1 shows the $\eta_{\mathrm{t} \text {,rce }}=0$ locations for the off-equatorial forcing maximum cases (cf. Hill et al. 2019; the $M_{\mathrm{t}, \text { rce }}<0$ and $f \eta_{\mathrm{t} \text {,rce }}<0$ conditions are never met in the $\varphi_{\mathrm{m}}=0$ case). For $\varphi_{\mathrm{m}}=6^{\circ}$, westerlies occurring just poleward of $\varphi_{\mathrm{m}}$ are sufficiently strong as to generate a local $M_{\mathrm{t}, \text { rce }}$ maximum poleward of $\varphi_{\mathrm{m}}$ that slightly exceeds the local planetary angular momentum value with either tropopause treatment, and this maximum is nearly unchanged $\left(9.0^{\circ}\right.$ vs $\left.9.1^{\circ}\right)$ when the fixed $-T_{\mathrm{t}}$ assumption is introduced. For $\varphi_{\mathrm{m}}=23.5^{\circ}$, the local $M_{\mathrm{t}, \mathrm{rce}}$ maximum is generated equatorward of $\varphi_{\mathrm{m}}$ at a value less than the local planetary angular momentum value, driven by strong easterlies, and this moves from $14.3^{\circ}$ to $14.8^{\circ}$ when a fixed $T_{\mathrm{t}}$ is assumed.

What explains this qualitative insensitivity of the Hide's theorem metrics to the tropopause meridional structure? For the $\varphi_{\mathrm{m}}=0^{\circ}$ case, $u_{\mathrm{t}, \text { rce }}$ is only altered by up to $\sim 3 \mathrm{~m} \mathrm{~s}^{-1}$, making the insensitivity unsurprising. But even if the differences were larger in magnitude, the $M_{\mathrm{t}, \text { rce }}=\Omega a^{2}$ point occurs within a few degrees latitude of where the fixed- $T_{\mathrm{t}}$ tropopause height goes from being greater than to less than its tropical-mean value $\overline{H_{\mathrm{t}}}$ used 
TABLE 1. Metrics of Hadley cell extent from Hide's theorem, the angular-momentum-conserving model, and the equal-area model (degrees latitude) and, for the equal-area model, the predicted depth-averaged potential temperature at the ascent latitude $\hat{\theta}_{a}$ (K) computed using either the traditional, fixed-height equations, or the new fixed-temperature equations. Also shown in parentheses next to each forcing maximum latitude value is $\overline{H_{\mathrm{t}}}$, i.e., the value used for the fixed- $H_{\mathrm{t}}$ calculations, namely, the tropopause height averaged over $30^{\circ} \mathrm{S}-30^{\circ} \mathrm{N}$ given that forcing and assuming a fixed $T_{\mathrm{t}}$.

\begin{tabular}{|c|c|c|c|c|c|c|}
\hline \multirow[b]{3}{*}{ Metric } & \multicolumn{6}{|c|}{$\varphi_{\mathrm{m}}\left(\overline{H_{\mathrm{t}}}\right)$} \\
\hline & \multicolumn{2}{|c|}{$0^{\circ}(10.4 \mathrm{~km})$} & \multicolumn{2}{|c|}{$6^{\circ}(10.4 \mathrm{~km})$} & \multicolumn{2}{|c|}{$23.5^{\circ}(9.7 \mathrm{~km})$} \\
\hline & Fixed $H_{\mathrm{t}}$ & Fixed $T_{\mathrm{t}}$ & Fixed $H_{\mathrm{t}}$ & Fixed $T_{\mathrm{t}}$ & Fixed $H_{\mathrm{t}}$ & Fixed $T$ \\
\hline$M_{\mathrm{t}, \text { rce }}>\Omega a^{2}$, summer & $15.5^{\circ}$ & $15.5^{\circ}$ & $10.0^{\circ}$ & $10.6^{\circ}$ & - & - \\
\hline$M_{\mathrm{t}, \text { rce }}>\Omega a^{2}$, winter & $-15.5^{\circ}$ & $-15.5^{\circ}$ & $-17.6^{\circ}$ & $-17.4^{\circ}$ & $-20.9^{\circ}$ & $-19.7^{\circ}$ \\
\hline$M_{\mathrm{t}, \mathrm{rce}}<0$ & - & - & $0.8^{\circ}$ & $0.8^{\circ}$ & $2.9^{\circ}$ & $3.1^{\circ}$ \\
\hline$f \eta_{\mathrm{t}, \mathrm{rce}}<0$ & - & - & $9.0^{\circ}$ & $9.1^{\circ}$ & $14.3^{\circ}$ & $14.8^{\circ}$ \\
\hline$u_{\mathrm{t}, \mathrm{rce}}=u_{\mathrm{amc}}$, summer & $15.5^{\circ}$ & $15.5^{\circ}$ & $22.3^{\circ}$ & $22.5^{\circ}$ & $35.4^{\circ}$ & $34.8^{\circ}$ \\
\hline$u_{\mathrm{t}, \mathrm{rce}}=u_{\mathrm{amc}}$, winter & $-15.5^{\circ}$ & $-15.5^{\circ}$ & $-24.7^{\circ}$ & $-24.3^{\circ}$ & $-38.7^{\circ}$ & $-36.7^{\circ}$ \\
\hline Equal-area $\varphi_{\mathrm{s}}$ & $19.5^{\circ}$ & $18.7^{\circ}$ & $24.1^{\circ}$ & $24.4^{\circ}$ & $35.9^{\circ}$ & $35.3^{\circ}$ \\
\hline Equal-area $\varphi_{\mathrm{w}}$ & $-19.5^{\circ}$ & $-18.7^{\circ}$ & $-32.7^{\circ}$ & $-32.5^{\circ}$ & $-51.1^{\circ}$ & $-48.7^{\circ}$ \\
\hline Equal-area $\varphi_{\mathrm{a}}$ & $0^{\circ}$ & $0^{\circ}$ & $18.1^{\circ}$ & $18.2^{\circ}$ & $34.5^{\circ}$ & $33.8^{\circ}$ \\
\hline Equal-area $\hat{\theta}_{\mathrm{a}}$ & $305.2 \mathrm{~K}$ & $305.2 \mathrm{~K}$ & $303.4 \mathrm{~K}$ & $303.3 \mathrm{~K}$ & $304.7 \mathrm{~K}$ & $304.8 \mathrm{~K}$ \\
\hline
\end{tabular}

in the fixed- $H_{\mathrm{t}}$ calculations (cf. Fig. 1). So $u_{\mathrm{t}, \mathrm{rce}}$, and in turn $M_{\mathrm{t}, \mathrm{rce}}$, is only modestly affected in the region of relevance. A similar phenomenon occurs for the $\eta_{\mathrm{t}, \mathrm{rce}}=$ 0 metric in the off-equator forcing cases. By construction, $u_{\mathrm{t}, \mathrm{rce}}=0$ at $\varphi_{\mathrm{m}}$ regardless of the tropopause structure, and at least for the cases examined the $\eta_{\mathrm{t}, \mathrm{rce}}=$ 0 point is not far removed from $\varphi_{\mathrm{m}}$. The effects of the tropopause treatment on $u_{\mathrm{t}, \mathrm{rce}}$, and in turn $M_{\mathrm{t} \text {,rce }}$ and $\eta_{\mathrm{t}, \text { rce }}$, in the vicinity are accordingly modest. In contrast, for $\varphi_{\mathrm{m}}=23.5^{\circ}$, the winter hemisphere $M_{\mathrm{t}, \mathrm{rce}}=\Omega a^{2}$ point occurs where the fixed- $T_{\mathrm{t}}$ tropopause height differs more appreciably from its tropical mean, yielding a nontrivial difference between the fixed-height versus fixed-temperature calculations.

We reiterate that all comparisons between fixed- $T_{\mathrm{t}}$ and fixed- $H_{\mathrm{t}}$ calculations depend on the $H_{\mathrm{t}}$ value chosen for the fixed- $H_{\mathrm{t}}$ calculations and that this choice is somewhat arbitrary. Nevertheless, as noted above, we deem the tropical-mean value from the fixed- $T_{\mathrm{t}}$ solutions, that is, $\overline{H_{t}}$, to be a reasonable choice-neither values much higher or much lower than $\overline{H_{\mathrm{t}}}$ seem justifiable.

\section{Effect of a fixed $T_{t}$ on the angular-momentum-conserving model}

Assuming that any of the three Hide's theorem conditions is met at any latitude (as can nearly always be expected; cf. Schneider 2006; Hill et al. 2019), the latitude-by-latitude RCE state cannot be sustained, and a Hadley circulation must emerge. Provided that drag is strong in the boundary layer but viscosity is small and eddy-induced stresses weak in the free troposphere, air parcels ascending out of the boundary layer will conserve the local planetary angular momentum value as they circulate within the free troposphere. If this ascent is concentrated into a narrow convergence zone at the latitude $\varphi_{\mathrm{a}}$ and vertical momentum advection is negligible (Shell and Held 2004; Adam and Paldor 2009), the Hadley cell will then exhibit the AMC zonal wind profile

$$
u_{\mathrm{amc}}=\Omega a \cos \varphi\left(\frac{\cos ^{2} \varphi_{\mathrm{a}}}{\cos ^{2} \varphi}-1\right)
$$

and a corresponding gradient-balanced AMC thermal field $\hat{\theta}_{\text {amc }}$ given by-if $H_{\mathrm{t}}$ is uniform-

$$
\frac{\hat{\theta}_{\mathrm{amc}}(\varphi)-\hat{\theta}_{\mathrm{a}}}{\theta_{0}}=-\frac{\Omega^{2} a^{2}}{2 g H_{\mathrm{t}}} \frac{\left(\cos ^{2} \varphi_{\mathrm{a}}-\cos ^{2} \varphi\right)^{2}}{\cos ^{2} \varphi},
$$

where $\hat{\theta}_{\mathrm{a}}$ is the value of $\hat{\theta}_{\mathrm{amc}}$ at $\varphi_{\mathrm{a}}$ (Held and Hou 1980; Lindzen and Hou 1988). At minimum, the resulting AMC Hadley circulation must span all latitudes where $u_{\text {amc }}<u_{\mathrm{t} \text {,rce }}$; otherwise a local extremum in $M_{\mathrm{t} \text {,rce }}$ would still exist at the cell edge in violation of Hide's theorem (Held and Hou 1980). Typically, this $u_{\mathrm{amc}}<u_{\mathrm{t}, \mathrm{rce}}$ expanse is broader than the minimal extent directly set by Hide's theorem.

In the previous sections relating to the hypothetical RCE state, the RCE thermal field was taken as given, and the task at hand was to determine how tropopause height variations alter the zonal wind field. For the AMC model, we consider the converse: the AMC wind $u_{\mathrm{amc}}$ varies only with latitude, not height, in thin-shell atmospheres (e.g., Fang and Tung 1996), and it is the columnaveraged temperature field in gradient balance with $u_{\text {amc }}$, $\hat{\theta}_{\text {amc }}$, that may be altered by a fixed $T_{\mathrm{t}}$. Strictly speaking, the original fixed- $T_{\mathrm{t}}$ argument (Seeley et al. 2019b) 

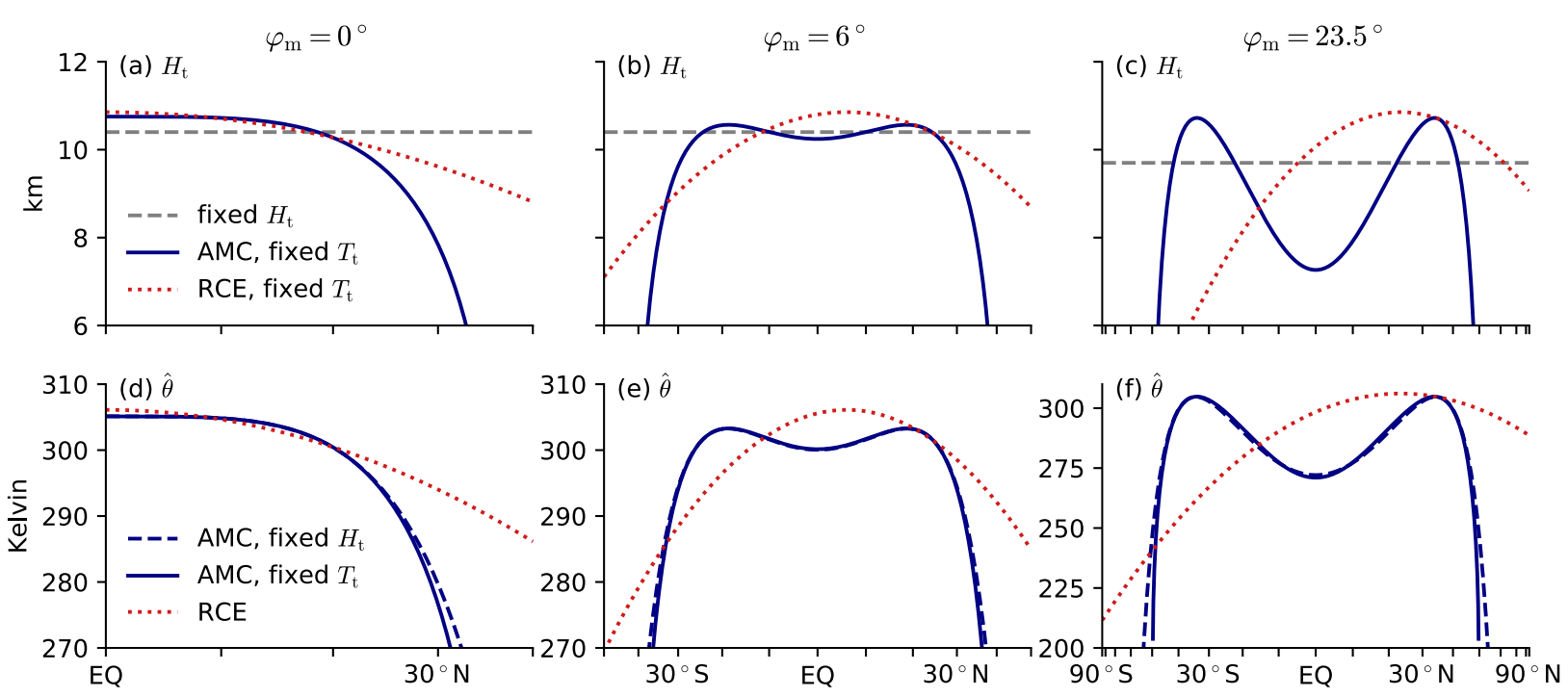

FIG. 3. (a)-(c) Tropopause height and (d)-(f) vertically averaged potential temperature corresponding to the RCE state given by (2), with the forcing maximum latitude $\varphi_{\mathrm{m}}$ set to (left to right) $0^{\circ}, 6^{\circ}$, or $23.5^{\circ}$, or to the angular-momentum-conserving (AMC) model, with the ascent latitudes used for the AMC solutions computed using the equal-area model applied to the given RCE potential temperature profile (listed in Table 1). Dashed lines indicate the tropopause height is assumed fixed; solid lines indicate the tropopause temperature is assumed fixed. Note differing horizontal-axis ranges in each column and differing vertical-axis ranges across panels in (d)-(f).

applies to RCE, not to the tropopause in the presence of a large-scale circulation. Nevertheless, in analogy to the assumption by Held and Hou (1980) of a uniform value of $H_{\mathrm{t}}$ within the AMC Hadley cells equal to its RCE value, we assume uniform values of $T_{\mathrm{t}}$ and $\hat{\Gamma}$ within the AMC Hadley cells equal to their RCE values.

Combining (1), (3), and (6) yields

$$
\frac{\partial \hat{\theta}}{\partial \varphi}=\frac{\theta_{0} \Omega^{2} a^{2}}{c_{p}\left(T_{\mathrm{s}}-T_{\mathrm{t}}\right)}\left(\frac{\sin \varphi}{\cos ^{3} \varphi} \cos ^{4} \varphi_{\mathrm{a}}-\cos \varphi \sin \varphi\right) .
$$

Bringing the $T_{\mathrm{s}}-T_{\mathrm{t}}$ term to the opposite side, using $\hat{\theta}=T_{\mathrm{s}}$ (recall that the stratification has been assumed dry adiabatic), and using the chain rule yields

$$
\frac{1}{2} \frac{\partial \hat{\theta}^{2}}{\partial \varphi}-T_{\mathrm{t}} \frac{\partial \hat{\theta}}{\partial \varphi}=\frac{\theta_{0} \Omega^{2} a^{2}}{c_{p}}\left(\frac{\sin \varphi}{\cos ^{3} \varphi} \cos ^{4} \varphi_{\mathrm{a}}-\cos \varphi \sin \varphi\right) .
$$

Integrating this expression in latitude and then applying the quadratic formula yields the AMC depth-averaged potential temperature for a fixed $T_{\mathrm{t}}$ and dry adiabatic lapse rate:

$\hat{\theta}_{\text {amc }}(\varphi)=T_{\mathrm{t}}+\sqrt{\left(\hat{\theta}_{\mathrm{a}}-T_{\mathrm{t}}\right)^{2}-\frac{\theta_{0} \Omega^{2} a^{2}\left(\cos ^{2} \varphi_{\mathrm{a}}-\cos ^{2} \varphi\right)^{2}}{c_{p}} \frac{\cos ^{2} \varphi}{}}$.

Figure 3 shows the RCE and AMC tropopause height and column-integrated potential temperature, with either fixed $H_{\mathrm{t}}$ or fixed $T_{\mathrm{t}}$ assumed, for each of the $\varphi_{\mathrm{m}}=0^{\circ}, 6^{\circ}$, $23.5^{\circ} \mathrm{RCE}$ cases discussed in previous sections. For each AMC solution, $\varphi_{\mathrm{a}}$ is determined from the equal-area model discussed in the next section. As was the case for the RCE state, a fixed $T_{\mathrm{t}}$ and lapse rate forces $H_{\mathrm{t}}$ to have the same meridional structure as $T_{\mathrm{s}}$, including the equatorial minimum for $\varphi_{\mathrm{a}} \neq 0$. In the $\varphi_{\mathrm{a}}=0$ case, this leads to a very flat tropopause height in low latitudes but a sharp shoulder poleward thereof $-H_{\mathrm{t}}$ decreases by $<0.5 \mathrm{~km}$ from the equator to $15^{\circ} \mathrm{N}$ and by $\sim 2.5 \mathrm{~km}$ over the subsequent $15^{\circ}$. For $\varphi_{\mathrm{m}} \neq 0$, this sharp shoulder emerges poleward of $\varphi_{\mathrm{a}}$ in the summer hemisphere and $-\varphi_{\mathrm{a}}$ in the winter hemisphere, occurring farther toward the midlatitudes as $\varphi_{\mathrm{m}}$ is increased.

These shoulders in $H_{\mathrm{t}}$, in turn, sharpen the corresponding shoulders of $\hat{\theta}_{\text {amc }}$. But ultimately this effect is modest (a few kelvins), and within the core of the Hadley cells the changes to $\hat{\theta}_{\text {amc }}$ are smaller still. The fixed- $T_{\mathrm{t}} \hat{\theta}_{\text {amc }}$ is undefined at values colder than $T_{\mathrm{t}}$, since this would imply a vanishing tropopause height.

Table 1 lists the locations of the $u_{\mathrm{amc}}=u_{\mathrm{t}, \text { rce }}$ points controlling the minimal AMC Hadley cell extent for each $\varphi_{\mathrm{m}}$ value $\left(u_{\mathrm{t}, \text { rce }}\right.$ profiles are shown in Fig. $2 \mathrm{a} ; u_{\mathrm{amc}}$ profiles are not shown). Recall that $\varphi_{\mathrm{m}}$ determines $u_{\mathrm{t}, \mathrm{rce}}$ and, via the equal-area model, $\varphi_{\mathrm{a}}$, and $\varphi_{\mathrm{a}}$ in turn determines $u_{\text {amc }}$, meaning that going from a fixed $H_{\mathrm{t}}$ to a fixed $T_{\mathrm{t}}$ can directly alter $u_{\mathrm{t}, \mathrm{rce}}$ and, through modifying $\varphi_{\mathrm{a}}$, indirectly alter $u_{\mathrm{amc}}$. Under annual-mean forcing, $\varphi_{\mathrm{a}}=\varphi_{\mathrm{m}}=0$ regardless of the tropopause treatment, and the $u_{\mathrm{amc}}=u_{\mathrm{t}, \mathrm{rce}}$ latitudes are essentially unchanged 
going from a fixed $H_{\mathrm{t}}$ to a fixed $T_{\mathrm{t}}$. For $\varphi_{\mathrm{m}}=6^{\circ}$, the equal-area-predicted $\varphi_{\mathrm{a}}$ is altered by only $0.1^{\circ}$ by the tropopause treatment. The summer hemisphere $u_{\text {amc }}=$ $u_{\mathrm{t}, \text { rce }}$ point is moved slightly poleward by a fixed $T_{\mathrm{t}}$ (by $0.2^{\circ}$ ), but its winter hemisphere counterpart contracts equatorward by slightly more, $0.4^{\circ}$. For $\varphi_{\mathrm{m}}=$ $23.5^{\circ}$, the equal-area-predicted $\varphi_{\mathrm{a}}$ is more sensitive to the tropopause treatment, being $34.5^{\circ}$ versus $33.8^{\circ}$ for a fixed height or temperature, respectively. This and the alterations to $u_{\mathrm{t}, \mathrm{rce}}$ cause the summer hemisphere $u_{\mathrm{t}, \mathrm{rce}}=u_{\mathrm{amc}}$ point to contract from $35.4^{\circ}$ to $34.8^{\circ}$ and the corresponding winter hemisphere point to contract from $-38.7^{\circ}$ to $-36.7^{\circ}$.

\section{Effect of a fixed $T_{t}$ on the equal-area model}

The equal-area model assumes that the Hadley cells conserve $^{5}$ angular momentum, that column-averaged temperature is continuous at all cell edges (which requires the Hadley cell temperatures to equal the RCE temperatures at the circulation's outer edges), and that the cells conserve energy in the sense that the potential temperature integrated over the extent of each cell is the same as it was over that extent in the RCE state (Held and Hou 1980; Lindzen and Hou 1988). It predicts, given only the knowledge of the RCE potential temperature distribution, the locations of all three Hadley cell edges (the two poleward edges and $\varphi_{\mathrm{a}}$ ) and $\hat{\theta}_{\mathrm{a}}$.

Figure 4 shows the equal-area model solutions for the three cell edges for $\varphi_{\mathrm{m}}$ varied from $0.1^{\circ}$ up to $23.5^{\circ}$, with the AMC temperature fields constructed either using (7) (in all cases with $\overline{H_{\mathrm{t}}}=10 \mathrm{~km}$ ) or (8) (with $T_{\mathrm{t}}=$ $200 \mathrm{~K}$ ), and with all other parameters Earthlike. And Table 1 lists these values and the corresponding $\hat{\theta}_{\mathrm{a}}$ solutions for the $\varphi_{\mathrm{m}}=0^{\circ}, 6^{\circ}, 23.5^{\circ}$ cases. Because going from fixed $H_{\mathrm{t}}$ to fixed $T_{\mathrm{t}}$ tends to sharpen the subtropical shoulder in $\hat{\theta}_{\text {amc }}$, the equal-area solutions for the cell poleward edges typically move equatorward, the more so the farther-poleward $\varphi_{\mathrm{m}}$ is and more so in the winter hemisphere than summer hemisphere (cf. Table 1, for $\varphi_{\mathrm{m}}=6^{\circ}$ the summer hemisphere edge actually moves slightly poleward). And $\varphi_{\mathrm{a}}$ moves either very weakly $\left(\varphi_{\mathrm{m}}=6^{\circ}\right)$ or modestly equatorward $\left(\varphi_{\mathrm{m}}=23.5^{\circ}\right)$. Though

\footnotetext{
${ }^{5}$ In the "strict" sense as in the preceding section, wherein the entire free-tropospheric expanse of the Hadley circulation possesses a single value of angular momentum. The term "angularmomentum-conserving" is sometimes used in a weaker sense in reference to states in which Hadley cell streamlines and angularmomentum contours are (approximately) coincident but the angular momentum nevertheless varies appreciably across the cells in the free troposphere.
}

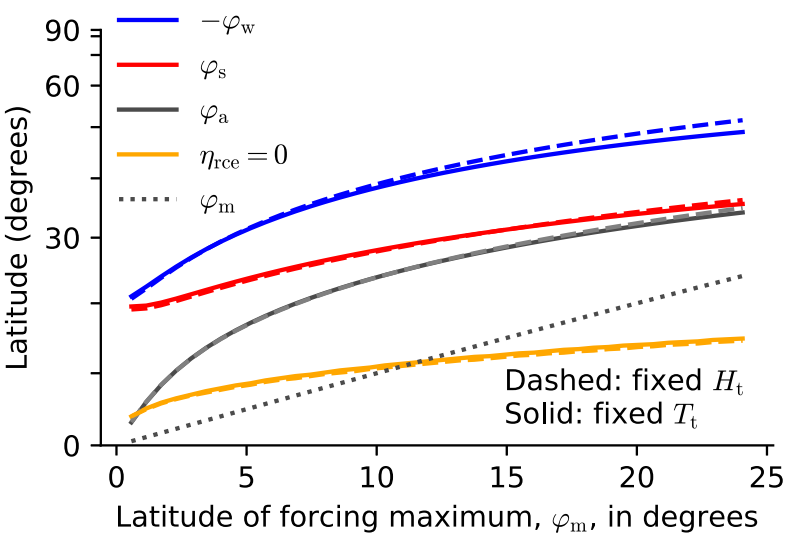

FIG. 4. Equal-area model solutions for the latitude of the poleward edges of the winter $\left(\varphi_{\mathrm{w}}\right.$; flipped in sign for easier visual comparison with the other metrics) and summer $\left(\varphi_{\mathrm{s}}\right)$ Hadley cells and their shared edge $\left(\varphi_{\mathrm{a}}\right)$, for $\varphi_{\mathrm{m}}$ ranging from $0.5^{\circ}$ to $23.5^{\circ}$. Dashed lines indicate $\hat{\theta}_{\text {amc }}$ is derived with the tropopause height assumed constant; solid lines indicate $\hat{\theta}_{\text {amc }}$ is derived with the tropopause temperature assumed constant. Orange curves show the latitude in the summer hemisphere where $\eta_{\mathrm{t}, \mathrm{rce}}=0$. Note that both the horizontal- and vertical-axis spacings are linear in sine of latitude.

the three predicted cell edges all move equatorward when comparing fixed- $H_{\mathrm{t}}$ to fixed- $T_{\mathrm{t}}$ calculations (for sufficiently large $\varphi_{\mathrm{m}}$ ), the equal-area predicted Hadley cell extents are still appreciably larger than observed and simulated solsticial Hadley cell extents for Earth (Hill et al. 2019).

Also overlaid in Fig. 4 are the $\eta_{\mathrm{t} \text {,rce }}=0$ locations assuming either fixed $H_{\mathrm{t}}$ to fixed $T_{\mathrm{t}}$ in Fig. 4. As $\varphi_{\mathrm{m}}$ moves poleward and the overall predicted Hadley circulation grows, $\eta_{\mathrm{t} \text {,rce }}=0$ falls equatorward of $\varphi_{\mathrm{m}}$ and becomes increasingly separated from $\varphi_{\mathrm{a}}$ (Hill et al. 2019), and this is insensitive to the tropopause treatment.

\section{Numerically simulated and analytically approximated latitude-by-latitude RCE}

The previous sections indicate that the results of nearly inviscid, axisymmetric Hadley cell theory are largely insensitive to how the tropopause height varies with latitude, at least under the given analytical profiles of the RCE thermal state. In principle, however, the tropopause treatment could be more influential when a more realistic latitude-by-latitude RCE state is considered. And, as described in the Introduction, the accuracy of the fixed- $T_{\mathrm{t}}$ assumption as applied on a latitude-bylatitude basis requires additional consideration. This section addresses those concerns.

\section{a. Model and simulations description}

We use the single-column model provided by the climlab package (Rose 2018) for the Python programming 
language. Convection is represented by simple convective adjustment (Manabe and Wetherald 1967) to a specified lapse rate that we set to 0.65 times the dry adiabat. Radiative transfer calculations use the RRTMG model (Mlawer et al. 1997) assuming clearsky conditions and a uniform surface albedo of 0.3 . Relative humidity is prescribed, using the profile of Manabe and Wetherald [1967, see their Eq. (2)], which decreases in height from a surface value of 0.77 but is uniform horizontally and in time. The surface is a mixed layer ocean of $1-\mathrm{m} \mathrm{depth} .^{6}$ There are no surface turbulent fluxes; instead convective adjustment adjusts both atmospheric and surface temperatures, with the surface time scale dependent on the mixedlayer depth. This setup is very similar to the singlecolumn model used by Dacie et al. (2019) in their study of the tropical tropopause layer.

Simulations are performed with time-invariant insolation representing present-day, annual-mean insolation sampled at latitude values separated by $1^{\circ}$, from $89.5^{\circ} \mathrm{S}$ to $89.5^{\circ} \mathrm{N}$. Each is run for 3000 days, with averages taken over the last 2200 days, wherein a steady state has been reached (Cronin and Emanuel 2013). Each column has 100 evenly spaced pressure levels from the surface to $0 \mathrm{hPa}$. We emphasize that each latitude is its own singlecolumn simulation with no communication across latitudes. The latitude value for each simulation is used to specify the insolation only; the single-column model does not account for rotational effects and is thus agnostic to the local Coriolis parameter.

From the equilibrated temperature profiles in each column, the tropopause is computed using several different metrics: the World Meteorological Organization definition of the lowest point at which the lapse rate reaches $2 \mathrm{~K} \mathrm{~km}^{-1}$ (WMO 1957); the "cold-point tropopause," that is, the coldest point in each column; and, cf. Schneider and Walker (2006), the location where the vertical curvature in temperature, that is, $\partial_{z z} T$, maximizes. A fixed-height tropopause definition is also computed using a value of $14 \mathrm{~km}$, a value chosen by eye to be similar within the tropics to the other tropopause definitions (results are insensitive to reasonable modifications of this value; not shown). On similarly ad hoc grounds, we select the $214 \mathrm{~K}$ isotherm as the fixed-temperature tropopause; values closer to the $200 \mathrm{~K}$ used in the preceding analytical work yields too deep a tropopause in the tropics compared to the other definitions (not shown).

\footnotetext{
${ }^{6}$ A smaller mixed-layer depth would lead to faster equilibration times, but at smaller depth instabilities can arise in highly insolated columns that cause the model to crash.
}

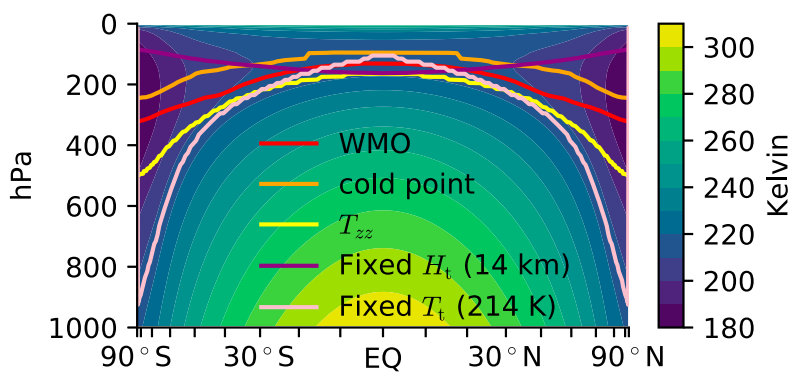

FIG. 5. Shading shows the temperature in the single-column model simulations of latitude-by-latitude RCE, with values as indicated in the color bar. The overlaid thick contours are various definitions of the tropopause, with colors corresponding to the legend.

To remove the distracting influence of gridpoint noise, we apply a 1-2-1 smoothing filter in latitude to the temperature field. Because the insolation and other boundary conditions are symmetric about the equator, we average the model output at each latitude across the Northern and Southern Hemispheres after applying the smoothing but before computing the various tropopause definitions or the gradient wind.

\section{b. Results}

Figure 5 shows the simulated equilibrium temperature distribution as a function of latitude and pressure with the tropopause indicators overlaid. All become colder overall going from the equator to the poles. Unlike all the other tropopause definitions, the pressure level of the fixed-height tropopause is highest in the tropics. All others generally become colder and sit at higher pressures moving poleward and are oriented with the curvature-based value below the WMO value, both of which sit somewhere below the cold point $(\mathrm{Hu}$ and Vallis 2019).

Qualitatively, the fixed- $T_{\mathrm{t}}$ assumption is largely borne out, in the sense that the given isotherm remains in the vicinity of the other, more conventional tropopause definitions from the equator to midlatitudes. And the other tropopause definitions all occur at temperatures within $210-220 \mathrm{~K}$ within $30^{\circ} \mathrm{S}-30^{\circ} \mathrm{N}$. At the same time, the fixed- $T_{\mathrm{t}}$ definition has the sharpest meridional drop of all, with near the highest values of all the definitions at the equator, dropping to the lowest in the midlatitudes, and then further dropping precipitously, nearly to the surface, at the poles.

Solid curves in Fig. 6 show $u_{\mathrm{t}, \mathrm{rce}}$ as a function of latitude diagnosed at each of these tropopause definitions, computed using the expression for gradient balance in pressure coordinates. Assuming negligible surface wind, this is 


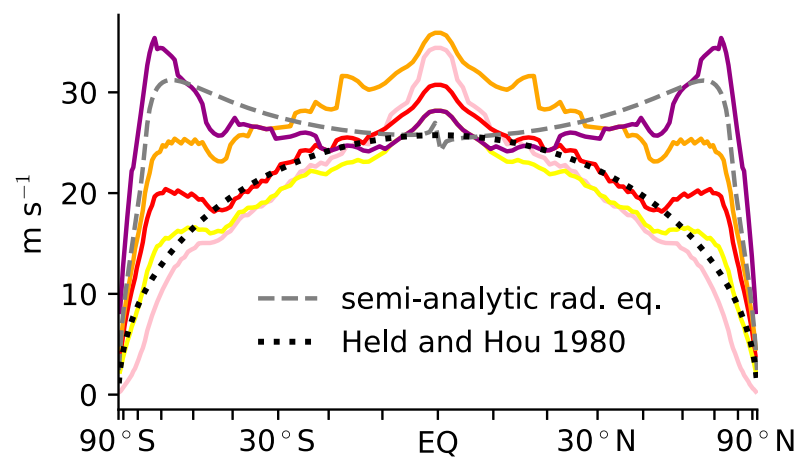

FIG. 6. Solid curves show $u_{\mathrm{t}, \mathrm{rce}}$ computed using the temperature structure from the single-column model simulations of latitude-bylatitude RCE shown in Fig. 5, assuming negligible surface wind, and using various definitions of the tropopause as indicated by the legend in Fig. 5. The dashed gray curve shows the semianalytical, radiativeequilibrium solution for surface temperature described in the main text, a tropopause height of $10 \mathrm{~km}$, and a lapse rate of $\Gamma=\gamma \Gamma_{\mathrm{d}}$ with $\gamma=0.65$ (small local maxima and minima straddling the equator are finite-differencing artifacts). The dotted black curve is the $u_{\mathrm{t}, \mathrm{rce}}$ distribution from Held and Hou (1980) (in its original, fixed- $H_{\mathrm{t}}$ form) using $\Delta_{\mathrm{h}}=1 / 8$ and $H_{\mathrm{t}}=10 \mathrm{~km}$.

$$
\begin{aligned}
& u(p, \varphi) \\
& =\Omega a \cos \varphi\left[\sqrt{1-\frac{1}{\cos \varphi \sin \varphi} \frac{R_{\mathrm{d}}}{\Omega^{2} a^{2}} \ln \left(\frac{p_{0}}{p}\right) \frac{\partial \hat{T}}{\partial \varphi}}-1\right]
\end{aligned}
$$

where $\hat{T}$ is the log-pressure-weighted average temperature from the surface pressure (set always to $p_{0}=$ $1000 \mathrm{hPa}$ ) to the given pressure $p .^{7}$

All share a common overall structure, maximizing near the equator and decreasing mostly monotonically poleward. Depending on the tropopause definition, the diagnosed $u_{\mathrm{t} \text {,rce }}$ value differs by as much $\sim 15 \mathrm{~m} \mathrm{~s}^{-1}$ at some low latitudes (and by more still at higher latitudes, but those are of less relevance to the Hadley cells). However, in the subtropics where the $M_{\mathrm{t}, \mathrm{rce}}>\Omega a^{2}$ condition becomes no longer satisfied, the $u_{\mathrm{t}, \text { rce }}$ (and in turn $M_{\mathrm{t}, \mathrm{rce}}$ ) differences across definitions are more modest: the $M_{\mathrm{t}, \mathrm{rce}}=\Omega a^{2}$ point lies between $12.5^{\circ}$ and $13.5^{\circ}$ for all of them except for the cold-point-based value, which is slightly farther poleward at $14.8^{\circ}$ (not shown).

\section{c. Nonnumerical approximations to simulation results}

We now consider two approximations to this simulated latitude-by-latitude RCE state that do not require

\footnotetext{
${ }^{7}$ Symbolically, $\hat{T} \equiv\left(\int_{\ln p}^{\ln p_{0}} T \mathrm{~d} \ln p\right) / \ln \left(p_{0} / p\right)$, a slight deviation from the previous notation in which hatted quantities corresponded to vertical averages (using the appropriate vertical coordinate) from the surface all the way to the tropopause.
}

running a numerical model. The first, which is purely analytic, is simply the original $\hat{\theta}_{\text {rce }}$ profile of Held and Hou (1980) - that is, that in gradient balance with (2) for $\varphi_{\mathrm{m}}=0$ - and the corresponding gradient-balanced zonal wind at the tropopause [see (5) of Held and Hou 1980]. To best fit the simulated latitudinal profile of depth-averaged temperature, we set $\Delta_{\mathrm{h}}$ to $1 / 8$ [smaller than the $1 / 3$ and $1 / 6$ values used by Held and Hou (1980) and Lindzen and Hou (1988), respectively]. In computing $u_{\mathrm{t}, \text { rce }}$, we retain the original $H_{\mathrm{t}, \mathrm{rce}}=10 \mathrm{~km}$ value of Held and Hou (1980). ${ }^{8}$

The second, which is semianalytical, applies the wellknown planetary energy balance (i.e., radiative equilibrium) model with a one-layer greenhouse at each latitude. Given the local top-of-atmosphere insolation $S$ and albedo $\alpha$, this yields a surface temperature of

$$
T_{\mathrm{s}}=2^{1 / 4}\left[\frac{S(1-\alpha)}{\sigma}\right]^{1 / 4}
$$

where $\sigma$ is the Stefan-Boltzmann constant. The corresponding $u_{\mathrm{t} \text {,rce }}$ field is then computed as follows. First, given $T_{\mathrm{s}}$ and $\Gamma=\gamma \Gamma_{\mathrm{d}}$ and neglecting any difference between surface pressure and the reference pressure appearing in the potential temperature (i.e., assuming $\left.p_{\mathrm{s}}=p_{0}\right)$, it follows that $\theta(z)=T_{\mathrm{s}}^{1 / \gamma}\left(T_{\mathrm{s}}-\gamma \Gamma_{\mathrm{d}} z\right)^{1-1 / \gamma}$, and therefore

$$
\hat{\theta}=\frac{\gamma}{2 \gamma-1} \frac{T_{\mathrm{s}}^{1 / \gamma}}{T_{\mathrm{s}}-T_{\mathrm{t}}}\left(T_{\mathrm{s}}^{2-1 / \gamma}-T_{\mathrm{t}}^{2-1 / \gamma}\right) .
$$

Using (9) at each latitude with $\gamma=0.65$ and the simple radiative-equilibrium-predicted $T_{\mathrm{s}}$, we compute the corresponding $\hat{\theta}$ distribution. We then compute $u_{\mathrm{t}, \mathrm{rce}}$ using (5) setting $\theta_{0}=290 \mathrm{~K}$ and, to be consistent with the Held and Hou (1980) approximation, $H_{\mathrm{t}, \mathrm{rce}}=10 \mathrm{~km}$.

Figure 7 shows, as deviations from their global average, the latitudinal distributions of troposphere-averaged temperature from the simulations (assuming a uniform tropopause pressure of $150 \mathrm{hPa}$ ) and potential temperature from the two approximations. Both approximations capture the overall meridional structure from the simulations, though with a steeper drop-off toward the poles, particularly for the radiative equilibrium solution.

The $u_{\mathrm{t}, \mathrm{rce}}$ fields computed using these two approximations are overlaid in Fig. 6 . The $u_{\mathrm{t}, \mathrm{rce}}$ values predicted

\footnotetext{
${ }^{8}$ These parameter values result in a thermal Rossby number, $R \equiv\left(g H_{\mathrm{t}, \mathrm{rce}} \Delta_{\mathrm{h}}\right) /\left(\Omega^{2} a^{2}\right)$, of $\sim 0.06$, lower than the original thermal Rossby number value from Held and Hou (1980) of $\sim 0.15$. If the latter is used, the resulting $u_{\mathrm{t}, \mathrm{rce}}$ values are too large over most of the domain, in particular being roughly $2-3$ times as large as the simulated values at low latitudes (not shown).
} 


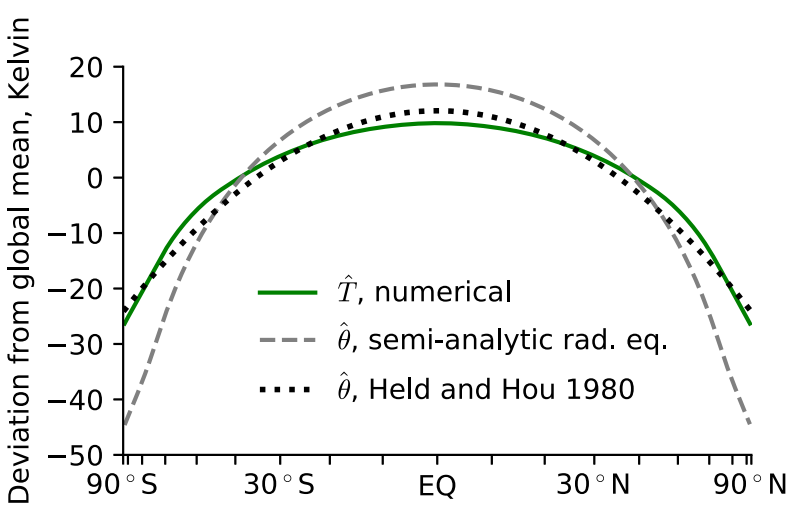

FIG. 7. The solid green curve shows temperature (K) from the single-column-model simulations averaged in pressure from the surface to the local tropopause (defined as occurring at a fixed pressure, $150 \mathrm{hPa}$, at all latitudes). The dashed gray and dotted black curves show the potential temperatures from the semianalytical, radiative-equilibrium approximation and from the original Held and Hou (1980) analytical expression for annual-mean forcing, respectively; both are averaged from the surface to a specified tropopause height of $10 \mathrm{~km}$. All three values are presented as a deviation from their respective global mean, since it is their meridional structure that influences $u_{\mathrm{t}, \text { rce }}$.

by both approximations (and as a result the corresponding $M_{\mathrm{t} \text {,rce }}$ values; not shown) fall within the range of the various diagnosed values at most latitudes, most importantly in the aforementioned $12.5^{\circ}-15.0^{\circ} \mathrm{N}$ range where the $M_{\mathrm{t} \text {,rce }}=\Omega a^{2}$ point occurs.

These results suggests that, at the level of accuracy presently considered, the properties of the latitude-bylatitude RCE state that acts as the "forcing" driving the Hadley cells can be interrogated without integrating a numerical model. Most usefully, they are captured adequately by the well-known analytical forcing from Held and Hou (1980) (with $\Delta_{\mathrm{h}}$ modified from $1 / 3$ to $1 / 8$ ).

\section{Summary}

Studies of axisymmetric Hadley cell theory-namely, those using Hide's theorem, the AMC model, and the equal-area model-have traditionally assumed that the tropopause height is uniform in latitude in the absence of an overturning circulation, i.e., in latitude-by-latitude RCE, and is unchanged by the emergence of the Hadley circulation. But recent work (Seeley et al. 2019b) suggests that the tropopause temperature is more nearly invariant than the tropopause height in RCE, at least on a domainmean basis. Insofar as the tropopause temperature and vertically averaged lapse rate do not vary meridionally, the tropopause height varies linearly with surface temperature, and the resulting meridional variations in tropopause height alter the gradient-balanced zonal wind at the tropopause that figures centrally in Hide's theorem, the AMC model, and the equal-area model. We rederive these axisymmetric theories assuming that the tropopause temperature is constant in latitude and across climate states. We then examine the tropopause temperature and Hadley cell metrics in numerical simulations of annual-mean, latitude-by-latitude RCE using a single-column model.

If the tropopause temperature is uniform, under standard annual-mean and solsticial Newtonian cooling reference temperature profiles (Lindzen and Hou 1988) the RCE tropopause height can vary in latitude by $\sim 40 \%$ across the tropics, being deepest where surface temperature is largest. Compared to the case if the tropopause height were uniform in latitude, the resulting gradient-balanced zonal wind at the tropopause can differ by up to tens of meters per second, but over most of the domain the differences are a few meters per second or less, particularly in the subtropics. Differences between fixed-height and fixed-temperature calculations are largest in the winter hemisphere for solsticial forcing and increase as the forcing maximum is moved toward the summer pole.

As such, the resulting absolute angular momentum and absolute vorticity fields, and with them the range of supercritical forcing based on the conditions of Hide's theorem, are weakly modified, by $\lesssim 1^{\circ}$. This is both because of the modest magnitudes of the changes to $u_{\mathrm{t}, \mathrm{rce}}$ and also because the Hide's theorem criteria typically stop being met in the subtropics precisely where the fixed-temperature tropopause height approaches its tropical-mean value that seems the most appropriate value to use for fixed-height calculations. In other words, where the values of $u_{\mathrm{t}, \mathrm{rce}}$ matter most for Hide's theorem, they are least sensitive to the tropopause treatment.

The AMC zonal wind $u_{\mathrm{amc}}$ is determined by the ascent latitude $\varphi_{\mathrm{a}}$ and is a function of latitude but not height (in thin-shell atmospheres like Earth's). It is thus unaltered by the tropopause treatment, but tropopause height variations consistent with a fixed $T_{\mathrm{t}}$ engender changes in the "critical" temperature field in gradient balance with that $u_{\text {amc }}$ field. Specifically, a sharp subtropical shoulder in $H_{\mathrm{t}}$ causes $\hat{\theta}_{\mathrm{amc}}$ to also have a sharper shoulder than does the fixed- $H_{\mathrm{t}}$ calculation. When plugged into the equal-area model, this results in a trivial to modest equatorward contraction of the overall predicted Hadley circulation extent. Qualitatively similar results for both the RCE and AMC quantities are found whether a dry or moist adiabat is assumed in the Boussinesq framework or if the CQE framework is used (appendixes A and B).

The tropopause temperature is reasonably uniform in latitude across the tropics in single-column model 
simulations sampling the full latitudinal range of Earth's annual-mean insolation. As was the case for the results using analytically specified RCE potential temperature profiles, the diagnosed $u_{\mathrm{t} \text {,rce }}$ field and with it the minimal Hadley cell extent from Hide's theorem is only modestly sensitive to the tropopause definition. Moreover, the simulated temperature fields and corresponding diagnosed $u_{\mathrm{t}, \text { rce }}$ fields are, given the purposes, adequately approximated by both a semianalytical approximation based on latitude-by-latitude radiative equilibrium and (with one parameter value modified from its standard value) the fully analytical forcing profile of Held and Hou (1980).

\section{Discussion}

We have assumed identical lapse rates and tropopause temperatures in RCE and in the presence of a large-scale overturning circulation. However, the Hadley cells must generate some positive static stability (dry in a dry atmosphere, moist in a moist atmosphere) that is not present in a true state of RCE in order to effect any net meridional energy transport and thus alter the depthaveraged temperature fields (Caballero et al. 2008). While this increased static stability going from RCE to the dynamically equilibrated state would not appear to alter the influence of the fixed- $T_{\mathrm{t}}$ assumption, it could in principle alter the equal-area solutions: at low latitudes, $\hat{\theta}$ will have increased overall, likely leading to a smaller meridional extent that conserves the $\hat{\theta}$ integral from the RCE state.

For moist atmospheres, some meridional variation in the vertically averaged lapse rate is likely over the extent of the Hadley cells, as can be seen from two largely independent lines of argument. First, cf. Held (2000), convection will be deepest where insolation and surface temperatures are highest, which combined with the weak temperature gradient (WTG) constraint at low latitudes (e.g., Sobel et al. 2001) sets the lapse rate remotely also. The resulting static stability suppresses convection in columns with lower insolation and surface temperature, ultimately producing an inversion at the boundary layer top. Second, cf. Emanuel (1995), if a significant fraction of a parcel's moisture is rained out in the ascending branch, then the parcel will be forced to warm dry adiabatically over most of its return to the surface in the descending branch, setting up a different lapse rate between the two branches and leading to a decoupling between the boundary layer and the free troposphere in the descending branch.

In the real tropics, a physically unambiguous tropopause level is not generally found; instead, the level where clear-sky radiative heating vanishes occurs below where the WMO lapse-rate definition is met, which in turn sits below the cold-point tropopause. The behavior of this tropical tropopause layer involves a rich interplay among radiative, dynamical (incorporating both the tropospheric circulations and the stratospheric Brewer-Dobson circulation), chemical, and thermodynamic processes (Fueglistaler et al. 2009). This makes temperature-based tropopause definitions less capable of demarcating the boundary between the dynamically active troposphere and comparatively quiescent stratosphere (Hu and Vallis 2019).

Moreover, in principle the depth of the Hadley circulation could separate from any of these other metrics. But one can argue that it should be closely related to the dynamical boundary between the troposphere and stratosphere: insofar as the majority of deep convection that penetrates to the tropopause occurs within the ITCZ as part of the zonal-mean Hadley cells, then the height of the Hadley circulation will indeed be the same height as the tropopause.

In response to mean surface warming, the temperature at which these metrics occur can rise nontrivially in GCMs (e.g., Vallis et al. 2015; Lin et al. 2017; Hu and Vallis 2019), in contrast to the RCE results in cloudresolving models, which are more climate-state invariant (Seeley et al. 2019b). Singh (2019) find the surfacetropopause temperature drop to be nearly invariant within the tropics in their idealized aquaplanet GCM featuring gray radiative transfer. Meanwhile, from Fig. 7 of $\mathrm{Hu}$ and Vallis (2019), one can infer that, in CMIP5 models in the first century of a standard $\mathrm{CO}_{2}$ doubling simulation (with $\mathrm{CO}_{2}$ increased by $1 \% \mathrm{yr}^{-1}$ until the initial value is doubled, in year 70), this surface-tropopause temperature difference increases markedly in high latitudes (where the surface warms strongly and the tropopause weakly) but least of all in the tropics (where, compared to higher latitudes, the surface warming is more moderate and tropopause warming more appreciable). Thus, to first order for the sake of the Hadley cells, a uniform $T_{\mathrm{s}}-T_{\mathrm{t}}$ value is reasonable, helping to explain that assumption's utility in the CQE framework.

The meridional structure of $H_{\mathrm{t}}$ in the fixed- $T_{\mathrm{t}}$, AMC solution for an on-equatorial forcing maximum bears resemblance to the real-world, annual-mean structure at sufficiently low latitudes (e.g., Fig. 1a of Hu and Vallis 2019), being quite flat over the deep tropics but with a sharp shoulder in the subtropics (though, of course, with the real-world shoulder then tapering off toward a more linear and gentle decrease in height moving farther poleward). This shoulder occurs farther poleward in observations (near $\sim 30^{\circ}$ ) than in the fixed- $T_{\mathrm{t}}$, AMC solution (closer to $\sim 20^{\circ}$ ). The poleward flanks of the annual-mean and equinoctial cells are known to be very 
strongly influenced by baroclinic eddy processes, and the AMC model is arguably inappropriate there, meaning that this similarity may not have much physical relevance for Earth. However, the fixed- $T_{\mathrm{t}}$, AMC shoulder may be of relevance to slower-rotating, smaller, or less strongly insolated planetary bodies (more formally, those with larger thermal Rossby numbers), since in such atmospheres baroclinicity is less important and axisymmetric processes more important. More generally, it is possible that sufficiently strong forcing or in planetary atmospheres sufficiently removed in parameter space from Earth's atmosphere, tropopause variations could make more of an impact than in the solutions we have presented for Earth under conventional forcings.

But for Earth's Hadley cells, these analytical results suggest that-notwithstanding concerns regarding the relevance of axisymmetric theory to Earth's macroturbulent, strongly eddying atmosphere (e.g., Schneider 2006) - the axisymmetric theory itself is quite insensitive to meridional tropopause variations. This should bolster (however modestly) our confidence in classical, dry, Boussinesq, nearly inviscid axisymmetric theory's utility: whatever its imperfections, assuming a fixed tropopause height is not an important one.

Acknowledgments. We thank two anonymous reviewers for catching several important mistakes in an earlier draft and for other helpful comments; addressing them greatly improved the manuscript. We thank Brian Rose for developing climlab and timely guidance in using it. S.A.H. was initially supported by NSF Atmospheric and Geospace Sciences Postdoctoral Research Fellowship (Award 1624740), and subsequently by the Caltech Foster and Coco Stanback Postdoctoral Fellowship. S.B. was supported by NSF Award AGS-1462544.

\section{APPENDIX A}

\section{Fixed- $T_{t}$, Non-Dry-Adiabatic Derivations and Results in the Boussinesq System}

In this appendix, we reproduce the relevant Boussinesq derivations from the main text but without the assumption of dry adiabatic stratification. We continue to assume a uniform lapse rate, but it now takes the form $\Gamma=\gamma \Gamma_{\mathrm{d}}$, with $\gamma<1$ representing dry-stable stratification.

Evaluating the integral on the right-hand side of (3) then requires the Leibniz integral rule, yielding

$$
\int_{0}^{H_{\mathrm{t}}(\varphi)} \frac{\partial \theta}{\partial \varphi} \mathrm{d} z=H_{\mathrm{t}} \frac{\partial \hat{\theta}}{\partial \varphi}-\left(\theta_{\mathrm{t}}-\hat{\theta}\right) \frac{\partial H_{\mathrm{t}}}{\partial \varphi},
$$

where $\theta_{\mathrm{t}}$ is potential temperature at the tropopause. After using (A1) in (3), the derivation for $u_{\mathrm{t}}$ proceeds as standard (i.e., by using the quadratic formula to solve for $u_{\mathrm{t}}$ ), resulting in

$$
u_{\mathrm{t}}=\Omega a \cos \varphi\left\{\sqrt{1-\frac{1}{\cos \varphi \sin \varphi} \frac{g}{\Omega^{2} a^{2} \theta_{0}}\left[H_{\mathrm{t}} \frac{\partial \hat{\theta}}{\partial \varphi}-\left(\theta_{\mathrm{t}}-\hat{\theta}\right) \frac{\partial H_{\mathrm{t}}}{\partial \varphi}\right]}-1\right\} .
$$

If $\gamma<1$, then $\theta_{\mathrm{t}}>\hat{\theta}$. Moreover, we expect the meridional gradients of $\hat{\theta}$ and $H_{\mathrm{t}}$ to have the same sign: the warmer the troposphere, the deeper the tropopause. Under these conditions, meridional tropopause height variations always act to reduce the magnitude of $u_{\mathrm{t}}$ compared to if $H_{\mathrm{t}}$ had the same value locally and no meridional gradient.

Even given a $\hat{\theta}_{\text {rce }}$ field, (A2) is not a closed expression unless $T_{\mathrm{s}}$ is known, which requires numerically solving (9) for $T_{\mathrm{s}}$. One could instead take the distribution of $T_{\mathrm{s}}$ as fixed and use it to solve for $\hat{\theta}$, but then the $\hat{\theta}_{\text {rce }}$ profile is no longer identical between the fixed- $H_{\mathrm{t}}$ and fixed- $T_{\mathrm{t}}$ cases, complicating the interpretation of any differences in the Hadley cell metrics between them.

The presence of the $\left(\theta_{\mathrm{t}}-\hat{\theta}\right) \partial_{\varphi} H_{\mathrm{t}}$ term in the gradient balanced $u_{\mathrm{t}, \text { rce }}$ for $\gamma<1$ makes a compact analytical solution for the fixed- $T_{\mathrm{t}} \hat{\theta}_{\text {amc }}$ field unattainable.
We conclude this appendix by comparing the fixed-lapserate, $\Gamma=\gamma \Gamma_{\mathrm{d}}$ formulation to the original vertical structure in $\theta_{\text {rce }}$ chosen by Held and Hou (1980) and Lindzen and Hou (1988). The latter is $\theta_{\text {rce }}(z) / \theta_{0}=\cdots+\Delta_{\mathrm{v}}\left(z / H_{\mathrm{t}}-1 / 2\right)$, where $\Delta_{\mathrm{v}}=1 / 8$ is an imposed fractional potential temperature increase from the surface to the tropopause. Assuming $p_{\mathrm{s}}=p(z=0)=p_{0}$, this implies a lapse rate of

$$
\frac{\mathrm{d} T}{\mathrm{~d} z}=\frac{\theta_{0} \Delta_{\mathrm{v}}}{H_{\mathrm{t}}}-\Gamma_{\mathrm{d}}\left[1+\frac{R_{\mathrm{d}}}{c_{p}} \ln \left(\frac{p_{0}}{p}\right)\right] .
$$

Using parameter values from Lindzen and Hou (1988), this yields effective $\gamma$ values ranging from roughly 0.7 to 0.9 , with the lapse rate decreasing vertically upward and poleward away from the forcing maximum (not shown). All else equal, vertically averaged lapse rates with $\gamma \approx 0.65$ would require $\Delta_{\mathrm{v}} \approx 1 / 4$ (not shown). 


\section{APPENDIX B}

\section{Fixed- $T_{t}$ Derivations and Results in the CQE System}

\section{a. Gradient balance}

The CQE derivation of gradient balance proceeds identically whether or not $T_{\mathrm{t}}$ is taken as fixed. Following Emanuel (1995), gradient balance in a CQE atmosphere may be expressed as

$$
\left.\frac{\partial T}{\partial p}\right|_{s^{*}} \frac{\partial s_{\mathrm{b}}}{\partial \varphi}=\frac{1}{a^{2}} \frac{\sin \varphi}{\cos ^{2} \varphi} \frac{\partial M^{2}}{\partial p},
$$

where $s^{*}$ is the saturation moist entropy and $s_{\mathrm{b}}=c_{p} \ln \theta_{\mathrm{eb}}$ the subcloud moist entropy with $\theta_{\mathrm{eb}}$ the subcloud equivalent potential temperature. The core assumption of (strict) CQE is that $s^{*}(p) \equiv s_{\mathrm{b}}$ at each latitude: convection is sufficiently frequent and vigorous as to make the time-mean stratification exactly moist adiabatic, with the time-mean saturation moist entropy at each height equal to the time-mean moist entropy of the subcloud air transported into the free troposphere by the convection. Integrating from the surface where $u \approx 0$ and thus $M \approx \Omega a^{2} \cos ^{2} \varphi$ to the tropopause yields

$$
\left(T_{\mathrm{s}}-T_{\mathrm{t}}\right) \frac{\partial s_{\mathrm{b}}}{\partial \varphi}=\frac{1}{a^{2}} \frac{\sin \varphi}{\cos ^{2} \varphi}\left(M^{2}-\Omega^{2} a^{4} \cos ^{4} \varphi\right) .
$$

Using the definitions of $s_{\mathrm{b}}$ and $M$, solving for $u_{\mathrm{t}, \text { rce }}$ yields $u_{\mathrm{t}, \mathrm{rce}}=\Omega a \cos \varphi\left[\sqrt{1-\frac{1}{\cos \varphi \sin \varphi} \frac{c_{p}\left(T_{\mathrm{s}}-T_{\mathrm{t}}\right)}{\Omega^{2} a^{2} \theta_{\mathrm{eb}}} \frac{\partial \theta_{\mathrm{eb}}}{\partial \varphi}}-1\right]$.

\section{b. Critical subcloud equivalent potential temperature field}

If $T_{\mathrm{s}}-T_{\mathrm{t}}$ is fixed, the CQE $\theta_{\mathrm{eb}}$ distribution in gradient balance with $u_{\mathrm{amc}}$ is, cf. (11) of Emanuel (1995),

$$
\theta_{\mathrm{eb}}=\theta_{\mathrm{eb}, \mathrm{a}} \exp \left[-\frac{\Omega^{2} a^{2}}{2 c_{p}\left(T_{\mathrm{s}}-T_{\mathrm{t}}\right)} \frac{\left(\cos ^{2} \varphi_{\mathrm{a}}-\cos ^{2} \varphi\right)^{2}}{\cos ^{2} \varphi}\right],
$$

where $\theta_{\mathrm{eb}, \mathrm{a}}$ is the value of $\theta_{\mathrm{eb}}$ at $\varphi_{\mathrm{a}}$.

Unlike for the gradient balance derivation, $T_{\mathrm{s}}$ must be specified in deriving the fixed- $T_{\mathrm{t}}, \mathrm{AMC} \theta_{\mathrm{eb}}$. On the one hand, the inclusion of moisture in the expression for $\theta_{\mathrm{e}}$ acts to make it larger than $T_{\mathrm{s}}$. On the other hand, we can expect a steeper-than-moist adiabatic lapse rate below cloud base due to the relative dearth of condensation.

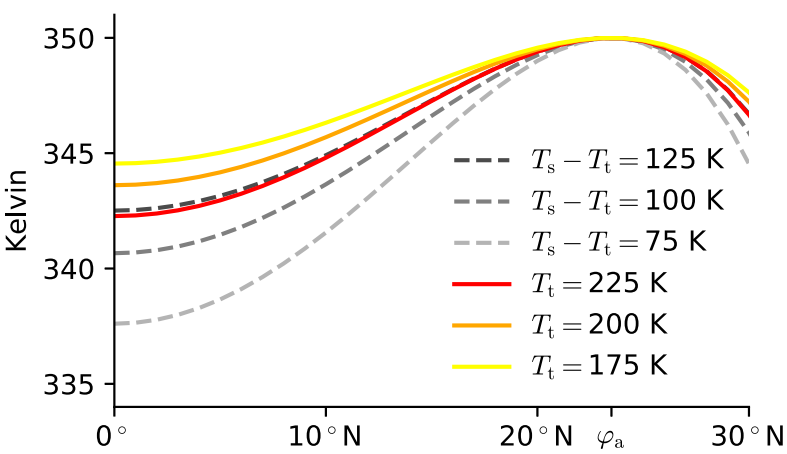

FIG. B1. Subcloud equivalent potential temperature profiles for which gradient wind balance yields AMC zonal wind at the tropopause in the CQE case, with an imposed maximum of $\theta_{\mathrm{eb}, \mathrm{a}}=350 \mathrm{~K}$ at $\varphi_{\mathrm{a}}=23.5^{\circ}$. The dashed curves assume a fixed tropospheric depth; the solid curves assume a fixed tropopause temperature and a surface temperature equal to the lowest-level potential temperature. Within each category, different colors correspond to different assumed values of the free parameter, according to the legend.

This acts to make $T_{\mathrm{s}}$ warmer than it would be if the stratification was moist adiabatic all the way to the surface-the more so the steeper the lapse rate or the deeper the subcloud layer. Given the level of approximation we are working at, these (at least partially) canceling influences lead us to simply take $\theta_{\mathrm{eb}} \approx T_{\mathrm{s}}$.

Under this assumption, the AMC $\theta_{\text {eb }}$ profile obeys

$\theta_{\mathrm{eb}}-T_{\mathrm{t}} \ln \frac{\theta_{\mathrm{eb}}}{\theta_{\mathrm{eb}, \mathrm{a}}}=\theta_{\mathrm{eb}, \mathrm{a}}-\frac{\Omega^{2} a^{2}}{2 c_{p}} \frac{\left(\cos ^{2} \varphi_{\mathrm{m}}-\cos ^{2} \varphi\right)^{2}}{\cos ^{2} \varphi}$.

This is not readily solvable analytically but is so numerically via standard root-finding algorithms (in this case, Brent's method as implemented in the SciPy package for the Python programming language).

Figure B1 shows critical potential temperature profiles for three different fixed tropospheric depths and with varying depth for three different tropopause temperatures, in all cases with $\varphi_{\mathrm{a}}=23.5^{\circ}$ and $\theta_{\mathrm{eb}, \mathrm{a}}=350 \mathrm{~K}$. By construction, all curves are equal to $\theta_{\mathrm{bm}}$ at $\varphi_{\mathrm{m}}$. The $\theta_{\mathrm{eb}}$ distribution attained with varying tropopause temperature has less meridional curvature at the equator and a weaker equator-to- $\varphi_{\mathrm{m}}$ gradient than the original, fixed-tropopause version. The default cases (orange solid and middle dashed curves) differ by roughly $3 \mathrm{~K}$ at the equator, with the fixed- $T_{\mathrm{t}}$ case warmer and thus with a smaller equator-to-maximum gradient.

\section{c. Relationship between Boussinesq and CQE results}

The exponential operator in (B4) arises from the use of entropy rather than potential temperature as the thermodynamic tracer (e.g., chapter 1 of Vallis 2017). A first-order Taylor expansion of (B4) yields 


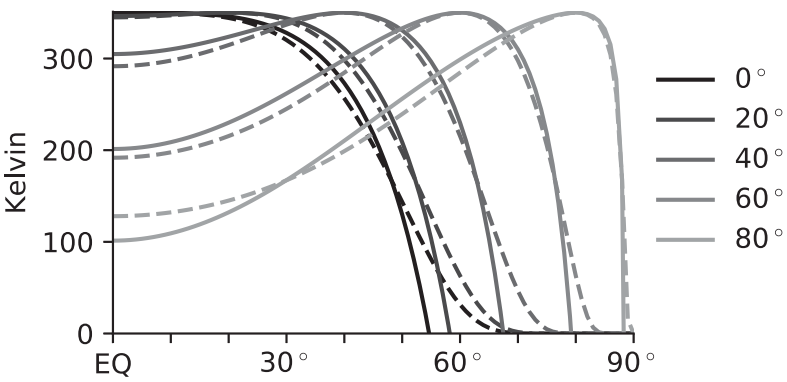

FIG. B2. Solid curves show Boussinesq $\hat{\theta}_{\text {amc }}$ fields given by (7) and dashed curves show CQE $\theta_{\mathrm{eb}, \mathrm{amc}}$ fields given by (B4). Pairs of curves with the same gray shading are the solutions for a given $\varphi_{\mathrm{a}}$, ranging from $0^{\circ}$ to $80^{\circ}$ in $20^{\circ}$ increments. In all cases the potential temperature value ( $\hat{\theta}_{\text {amc }}$ for Boussinesq, $\theta_{\mathrm{eb}, \mathrm{amc}}$ for CQE) at the maximum is set to $350 \mathrm{~K}$. For the CQE profiles, the difference between the surface and tropopause temperatures, $T_{\mathrm{s}}-T_{\mathrm{t}}$, is taken as a constant $100 \mathrm{~K}$. The free parameters of the Lindzen and Hou (1988) profiles were then chosen subjectively to minimize the differences between the Lindzen and Hou (1988) and Emanuel (1995) solutions. In particular, the assumed tropospheric depth is $12 \mathrm{~km}$, and the reference potential temperature is $290 \mathrm{~K}$. Note that, unlike figures in the main text, the horizontal spacing is linear in latitude, rather than sine of latitude, to make the high-latitude- $\varphi_{\mathrm{a}}$ curves more easily seen.

$$
\frac{\theta_{\mathrm{eb}}(\varphi)-\theta_{\mathrm{eb}, \mathrm{a}}}{\theta_{\mathrm{eb}, \mathrm{a}}} \approx-\frac{\Omega^{2} a^{2}}{2 c_{p}\left(T_{\mathrm{s}}-T_{\mathrm{t}}\right)} \frac{\left(\cos ^{2} \varphi_{\mathrm{a}}-\cos ^{2} \varphi\right)^{2}}{\cos ^{2} \varphi},
$$

Comparing (B6) and (7), we see that the Boussinesq $\hat{\theta}_{\text {amc }}$ amounts to a linearization about $\varphi_{\mathrm{a}}$ of its dry CQE counterpart.

This is demonstrated in Fig. B2, which shows (7) and (B4) over one hemisphere with forcing maxima of $0^{\circ}, 20^{\circ}, 40^{\circ}, 60^{\circ}$, and $80^{\circ} \mathrm{N}$, assuming $\theta_{\mathrm{eb}, \mathrm{a}}=350 \mathrm{~K}$ and $T_{\mathrm{s}}-T_{\mathrm{t}}=100 \mathrm{~K}$ in the Emanuel (1995) solution, and with the Lindzen and Hou (1988) parameters $H=12 \mathrm{~km}$ and $\theta_{0}=290 \mathrm{~K}$ chosen subjectively by eye to minimize the difference between the two models. Both versions are mirror symmetric about the equator, with $\sim \varphi^{4}$ dependence at the equator for $\varphi_{\mathrm{a}}=0^{\circ}$, thereby preventing equatorial westerlies (Schneider 2006; Hill et al. 2019). The Boussinesq solutions have the undesirable feature of passing through absolute zero, but the CQE cases likewise lose their physical meaningfulness once surface temperatures drop to sufficiently low values, certainly once $\theta_{\mathrm{eb}} \sim T_{\mathrm{t}}$.

\section{REFERENCES}

Adam, O., and N. Paldor, 2009: Global circulation in an axially symmetric shallow water model forced by equinoctial differential heating. J. Atmos. Sci., 66, 1418-1433, https://doi.org/ 10.1175/2008JAS2685.1

— and - 2010: Global circulation in an axially symmetric shallow-water model, forced by off-equatorial differential heating. J. Atmos. Sci., 67, 1275-1286, https://doi.org/10.1175/ 2009JAS3324.1.

Caballero, R., R. T. Pierrehumbert, and J. L. Mitchell, 2008: Axisymmetric, nearly inviscid circulations in non-condensing radiative-convective atmospheres. Quart. J. Roy. Meteor. Soc., 134, 1269-1285, https://doi.org/10.1002/qj.271.

Cronin, T. W., and K. A. Emanuel, 2013: The climate time scale in the approach to radiative-convective equilibrium. J. Adv. Model. Earth Syst., 5, 843-849, https://doi.org/10.1002/jame.20049.

Dacie, S., and Coauthors, 2019: A 1D RCE study of factors affecting the tropical tropopause layer and surface climate. J. Climate, 32, 6769-6782, https://doi.org/10.1175/JCLI-D18-0778.1.

Emanuel, K. A., 1995: On thermally direct circulations in moist atmospheres. J. Atmos. Sci., 52, 1529-1534, https://doi.org/ 10.1175/1520-0469(1995)052<1529:OTDCIM>2.0.CO;2.

_ J. D. Neelin, and C. S. Bretherton, 1994: On large-scale circulations in convecting atmospheres. Quart. J. Roy. Meteor. Soc., 120, 1111-1143, https://doi.org/10.1002/qj.49712051902.

Fang, M., and K. K. Tung, 1994: Solution to the Charney problem of viscous symmetric circulation. J. Atmos. Sci., 51, 1261-1272, https://doi.org/10.1175/1520-0469(1994)051<1261:STTCPO> 2.0.CO;2.

— , and - 1996: A simple model of nonlinear Hadley circulation with an ITCZ: Analytic and numerical solutions. J. Atmos. Sci., 53, 1241-1261, https://doi.org/10.1175/15200469(1996)053<1241:ASMONH>2.0.CO;2.

Fueglistaler, S., A. E. Dessler, T. J. Dunkerton, I. Folkins, Q. Fu, and P. W. Mote, 2009: Tropical tropopause layer. Rev. Geophys., 47, RG1004, https://doi.org/10.1029/2008RG000267.

Hartmann, D. L., and K. Larson, 2002: An important constraint on tropical cloud-climate feedback. Geophys. Res. Lett., 29, 1951, https://doi.org/10.1029/2002GL015835.

Held, I. M., 1982: On the height of the tropopause and the static stability of the troposphere. J. Atmos. Sci., 39, 412-417, https://doi.org/10.1175/1520-0469(1982)039<0412:OTHOTT $>$ 2.0.CO;2.

2000: The general circulation of the atmosphere. Woods Hole Oceanographic Institution Tech. Rep. WHOI-2001-03, 179 pp.

, and A. Y. Hou, 1980: Nonlinear axially symmetric circulations in a nearly inviscid atmosphere. J. Atmos. Sci., 37, 515-533, https:// doi.org/10.1175/1520-0469(1980)037<0515:NASCIA > 2.0.CO;2.

- and M. J. Suarez, 1994: A proposal for the intercomparison of the dynamical cores of atmospheric general circulation models. Bull. Amer. Meteor. Soc., 75, 1825-1830, https://doi.org/10.1175/ 1520-0477(1994)075<1825:APFTIO>2.0.CO;2.

Hide, R., 1969: Dynamics of the atmospheres of the major planets with an appendix on the viscous boundary layer at the rigid bounding surface of an electrically-conducting rotating fluid in the presence of a magnetic field. J. Atmos. Sci., 26, 841-853, https://doi.org/10.1175/1520-0469(1969)026<0841:DOTAOT> 2.0.CO;2.

Hill, S. A., S. Bordoni, and J. L. Mitchell, 2019: Axisymmetric constraints on cross-equatorial Hadley cell extent. J. Atmos. Sci., 76, 1547-1564, https://doi.org/10.1175/JAS-D-18-0306.1.

$\mathrm{Hu}, \mathrm{S}$., and G. K. Vallis, 2019: Meridional structure and future changes of tropopause height and temperature. Quart. J. Roy. Meteor. Soc., 145, 2698-2717, https://doi.org/10.1002/qj.3587.

Lin, P., D. Paynter, Y. Ming, and V. Ramaswamy, 2017: Changes of the tropical tropopause layer under global warming. J. Climate, 30, 1245-1258, https://doi.org/10.1175/JCLI-D-16-0457.1.

Lindzen, R. S., and A. V. Hou, 1988: Hadley circulations for zonally averaged heating centered off the equator. J. Atmos. Sci., 
45, 2416-2427, https://doi.org/10.1175/1520-0469(1988)045<2416: $\mathrm{HCFZAH}>2.0 . \mathrm{CO} ; 2$.

Manabe, S., and R. T. Wetherald, 1967: Thermal equilibrium of the atmosphere with a given distribution of relative humidity. J. Atmos. Sci., 24, 241-259, https://doi.org/10.1175/ 1520-0469(1967)024<0241:TEOTAW >2.0.CO;2.

Mlawer, E. J., S. J. Taubman, P. D. Brown, M. J. Iacono, and S. A. Clough, 1997: Radiative transfer for inhomogeneous atmospheres: RRTM, a validated correlated-k model for the longwave. J. Geophys. Res., 102, 16 663-16 682, https:// doi.org/10.1029/97JD00237.

Plumb, R. A., and A. Y. Hou, 1992: The response of a zonally symmetric atmosphere to subtropical thermal forcing: Threshold behavior. J. Atmos. Sci., 49, 1790-1799, https://doi.org/10.1175/ 1520-0469(1992)049<1790:TROAZS > 2.0.CO;2.

Richardson, M. I., and R. J. Wilson, 2002: A topographically forced asymmetry in the Martian circulation and climate. Nature, 416, 298-301, https://doi.org/10.1038/416298a.

Rose, B. E. J., 2018: CLIMLAB: A Python toolkit for interactive, process-oriented climate modeling. J. Open Source Software, 3, 659-660, https://doi.org/10.21105/joss.00659.

Schneider, E. K., 1977: Axially symmetric steady-state models of the basic state for instability and climate studies. Part II. Nonlinear calculations. J. Atmos. Sci., 34, 280-296, https://doi.org/10.1175/ 1520-0469(1977)034<0280:ASSSMO > 2.0.CO;2.

_ 1987: A simplified model of the modified Hadley circulation. J. Atmos. Sci., 44, 3311-3328, https://doi.org/10.1175/15200469(1987)044<3311:ASMOTM>2.0.CO;2.

Schneider, T., 2004: The tropopause and the thermal stratification in the extratropics of a dry atmosphere. J. Atmos. Sci., 61, 1317-1340, https://doi.org/10.1175/1520-0469(2004)061<1317: TTATTS $>2.0 . \mathrm{CO} ; 2$.

2006: The general circulation of the atmosphere. Annu. Rev. Earth Planet. Sci., 34, 655-688, https://doi.org/10.1146/ annurev.earth.34.031405.125144.

, and C. C. Walker, 2006: Self-organization of atmospheric macroturbulence into critical states of weak nonlinear eddyeddy interactions. J. Atmos. Sci., 63, 1569-1586, https:// doi.org/10.1175/JAS3699.1.

, and S. Bordoni, 2008: Eddy-mediated regime transitions in the seasonal cycle of a Hadley circulation and implications for monsoon dynamics. J. Atmos. Sci., 65, 915-934, https://doi.org/ 10.1175/2007JAS2415.1.
Seeley, J. T., N. Jeevanjee, W. Langhans, and D. M. Romps, 2019a: Formation of tropical anvil clouds by slow evaporation. Geophys. Res. Lett., 46, 492-501, https://doi.org/10.1029/ 2018 GL080747.

,-- , and D. M. Romps, 2019b: FAT or FiTT: Are anvil clouds or the tropopause temperature invariant? Geophys. Res. Lett., 46, 1842-1850, https://doi.org/10.1029/2018GL080096.

Shell, K. M., and I. M. Held, 2004: Abrupt transition to strong superrotation in an axisymmetric model of the upper troposphere. J. Atmos. Sci., 61, 2928-2935, https://doi.org/10.1175/ JAS-3312.1.

Singh, M. S., 2019: Limits on the extent of the solsticial Hadley cell: The role of planetary rotation. J. Atmos. Sci., 76, 1989-2004, https://doi.org/10.1175/JAS-D-18-0341.1.

Sobel, A. H., J. Nilsson, and L. M. Polvani, 2001: The weak temperature gradient approximation and balanced tropical moisture waves. J. Atmos. Sci., 58, 3650-3665, https://doi.org/ 10.1175/1520-0469(2001)058<3650:TWTGAA > 2.0.CO;2.

Thompson, D. W. J., S. Bony, and Y. Li, 2017: Thermodynamic constraint on the depth of the global tropospheric circulation. Proc. Natl. Acad. Sci. USA, 114, 8181-8186, https://doi.org/ 10.1073/pnas.1620493114.

— - P. Ceppi, and Y. Li, 2019: A robust constraint on the temperature and height of the extratropical tropopause. J. Climate, 32, 273-287, https://doi.org/10.1175/JCLI-D-18-0339.1.

Vallis, G. K., 2017: Atmospheric and Oceanic Fluid Dynamics: Fundamentals and Large-Scale Circulation. 2nd ed. Cambridge University Press, 946 pp.

_ P. Zurita-Gotor, C. Cairns, and J. Kidston, 2015: Response of the large-scale structure of the atmosphere to global warming. Quart. J. Roy. Meteor. Soc., 141, 1479-1501, https://doi.org/ 10.1002/qj.2456.

Walker, C. C., and T. Schneider, 2005: Response of idealized Hadley circulations to seasonally varying heating. Geophys. Res. Lett., 32, L06813, https://doi.org/10.1029/2004GL022304.

- , and - 2006: Eddy influences on Hadley circulations: Simulations with an idealized GCM. J. Atmos. Sci., 63, 33333350, https://doi.org/10.1175/JAS3821.1.

WMO, 1957: Meteorology-A three-dimensional science. WMO Bull., 6, 134-138.

Zalucha, A. M., R. A. Plumb, and R. J. Wilson, 2010: An analysis of the effect of topography on the Martian Hadley cells. J. Atmos. Sci., 67, 673-693, https://doi.org/10.1175/2009JAS3130.1. 